\title{
Approaches to monitor ATP levels in living cells: where do we stand?
}

\section{Seyta Ley-Ngardigal ${ }^{1}$ and Giulia Bertolin ${ }^{1, *}$}

${ }^{1}$ CNRS, Univ Rennes, IGDR (Genetics and Development Institute of Rennes), UMR 6290, F-35000 Rennes, France

* Correspondence: giulia.bertolin@univ-rennes1.fr; Tel: +330223237516

\begin{abstract}
ATP is the most universal and essential energy molecule in the eukaryotic cell. This is due to its ability to store energy in form of high energy phosphate bonds, which are extremely stable and readily usable by the cell. This energy is key for a variety of biological functions such as cell growth and division, metabolism, signalling, and for the turnover of biomolecules. Understanding how ATP is produced and hydrolysed with a spatiotemporal resolution is necessary to understand its functions both in physiological and pathological contexts. In this review, we will first describe the ATP synthase, the main molecular motor for ATP production in mitochondria. Second, we will review the biochemical assays currently available to estimate ATP quantities in cells, and we will compare their readouts, strengths and weaknesses. Then, we will explore the palette of genetically-encoded biosensors designed for microscope-based approaches and show how their spatiotemporal resolution opened up the possibility to follow ATP levels and production in living cells. Finally, we will comment on how ATP monitoring is used in preclinical practices, and to what extent genetically-encoded sensors could be used as a promising tool to elucidate pathologies in which ATP is implicated.
\end{abstract}

Keywords: ATP, OXPHOS, ATP synthase, mitochondria, biochemical assays, fluorescence microscopy, genetically-encoded biosensors. 


\section{ATP production and functioning of the ATP synthase}

ATP (or Adenosine Triphosphate) is a key and universal energy molecule with the ability to store and transport energy. This is achieved thanks to the phosphoanhydride bonds between the $\alpha-\beta$ phosphate groups, and between the $\beta-\gamma$ ones. These are defined as high energy bonds, because their hydrolysis is energetically favourable, and it liberates more than $7,2 \mathrm{kcal} / \mathrm{mole}$ [1,2]. The energy released from the hydrolysis of the phosphoanhydride bond is essential to almost all cellular processes and it is consumed to realise a various number of reactions. For example, the phosphorylation of 3-phosphoglycerate into 1,3-bisphosphoglycerate during gluconeogenesis consumes $4,8 \mathrm{kcal} / \mathrm{mole}$ [3]. Again, the $\mathrm{Na}^{+} / \mathrm{K}^{+}$pump allows the expulsion of 3 atoms of $\mathrm{Na}^{+}$and the entry of 2 atoms of $\mathrm{K}^{+}$into the cell by consuming 1 molecule of ATP, therefore approximately 7,2 $\mathrm{kcal} / \mathrm{mole}$ [4]. In processes regulating synaptic communication, the formation of vesicles filled with neurotransmitters and their release into the synaptic cleft consumes 1 molecule of ATP per vesicle $[5,6]$. Last, in muscular contraction, the sliding of the myosin head on the actin filament consumes 1 molecule of ATP as well [7]. Overall, cellular activities can be seen as large energy consuming processes and where ATP is the most common energy source [2,8]. In addition to its energy-providing role, ATP is a key molecule involved in cell signalling [9], and particularly in purinergic signaling [8]. Indeed, the ATP molecule has the ability to be recognized by purinergic receptors, and it plays key roles in inflammation [10,11], in the coagulation response, in cell proliferation, in synaptic transmission, and in cell permeability among other roles $[12,13]$.

From a structural point of view, there are significant electrostatic repulsions between the negatively-charged phosphate groups within one molecule of ATP. As a result, ATP tends to undergo spontaneous hydrolysis. Because of this feature, ATP (as well as ADP, Adenosine Diphosphate) is found in a complex with the magnesium ion $\left(\mathrm{Mg}^{2+}\right)$ within the cell. The $\mathrm{Mg}^{2+}$-ATP complex reduces the electrostatic repulsions within the ATP molecule, due to the positive charges of the $\mathrm{Mg}^{2+}$ ion that partially neutralize the negative charges of oxygen. This makes the $\mathrm{Mg}^{2+}$-ATP complex less prone to spontaneous hydrolysis, thus more stable in the cell than ATP in its free form [14] (Figure 1).

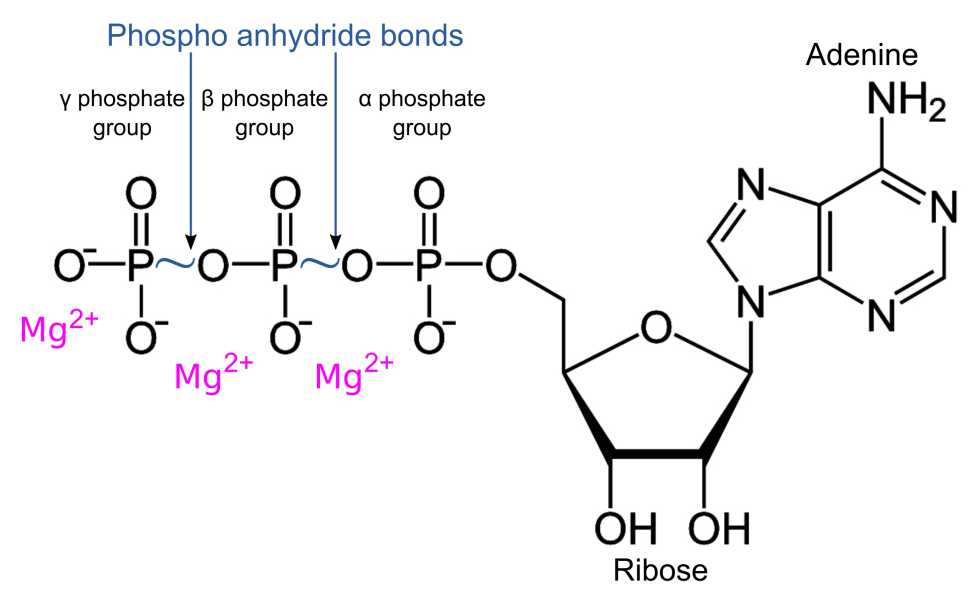

Figure 1. Chemical representation of ATP structure. ATP is a nucleotide composed of adenine (purine base), ribose (pentose) and of 3 phosphate groups. Within the cell, ATP is stabilized by $\mathrm{Mg}^{2+}$ in order to form $\mathrm{Mg}^{2+}-\mathrm{ATP}^{-}$ complex.

Due to the multiple and pivotal roles of ATP, the eukaryotic cell established multiple pathways to synthesize this essential molecule. The energy-providing biomolecules are carbohydrates, lipids and proteins, and are mostly obtained with food intake. These molecules allow ATP to be synthesized by different catabolic pathways as glycolysis, lipolysis and proteolysis respectively. Each of these pathways converges on a common pathway: oxidative phosphorylation, the main route for aerobic ATP synthesis [15] (Figure 2). 

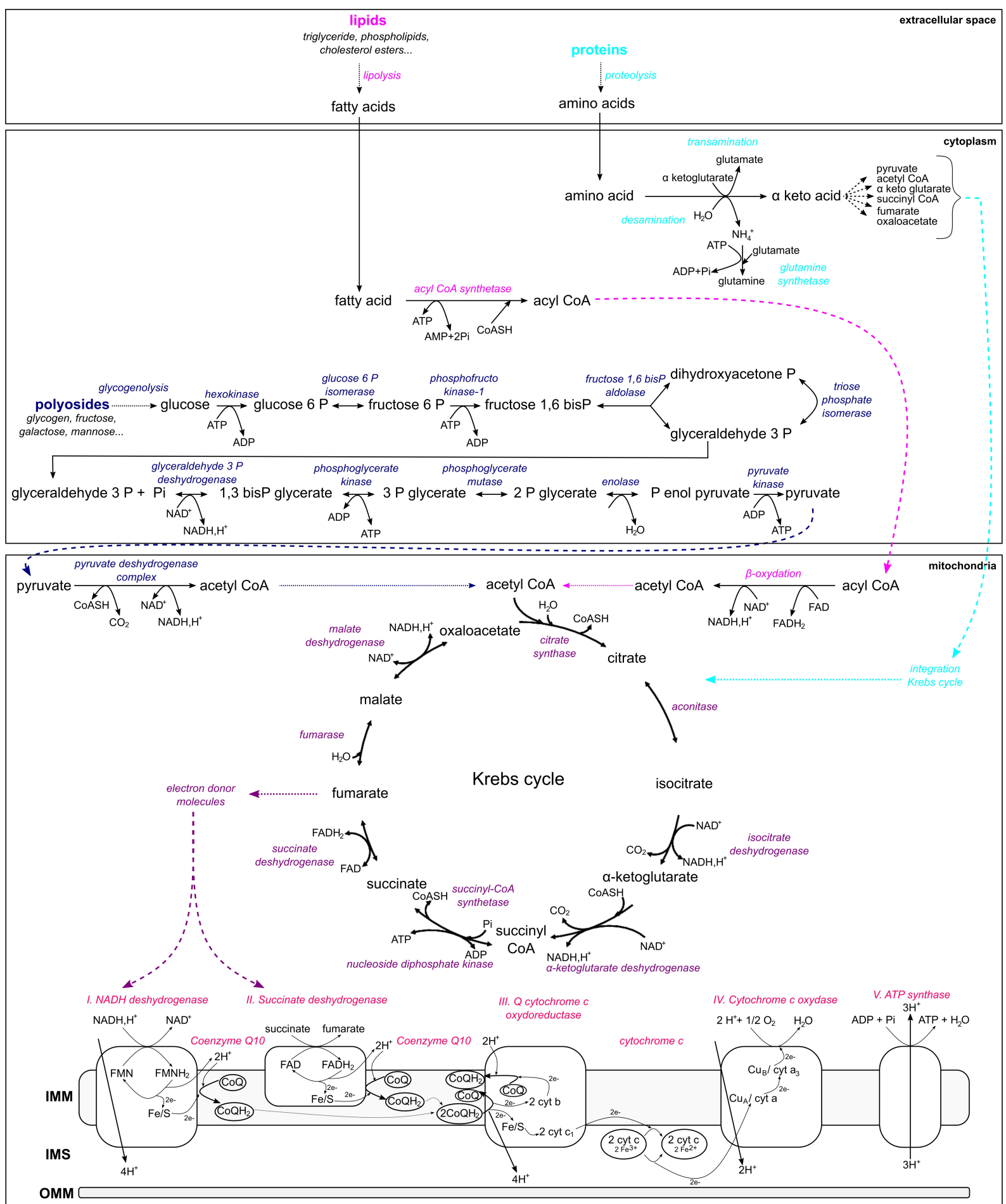

Figure 2. Metabolic pathways leading to ATP synthesis

This figure shows the degradation of lipids by lipolysis (magenta), proteins by proteolysis (cyan) and carbohydrates by glycolysis (blue). These three pathways converge on the Krebs cycle (purple), followed by oxidative phosphorylation (pink) to form ATP. IMM corresponds to Inner Mitochondrial Membrane, IMS corresponds to Intermembane Mitochondrial Space.

In the sections below, we will focus on the comparison between the bacterial and eukaryotic complexes orchestrating oxidative phosphorylation, and on structural insights of the ATP synthase. 


\subsection{Oxidative phosphorylation}

Oxidative phosphorylation is the process coupling the oxidation of electron-donor molecules $\mathrm{NADH}, \mathrm{H}^{+}$and $\mathrm{FADH}_{2}$ - with the phosphorylation of ADP into ATP. This process takes place at the level of the inner mitochondrial membrane, within the mitochondrial respiratory chain. Functionally speaking, the mitochondrial respiratory chain can be divided into the electron transport chain module (complexes I to IV) and the ATP synthase (Complex V) (Figure 2, Figure 3).

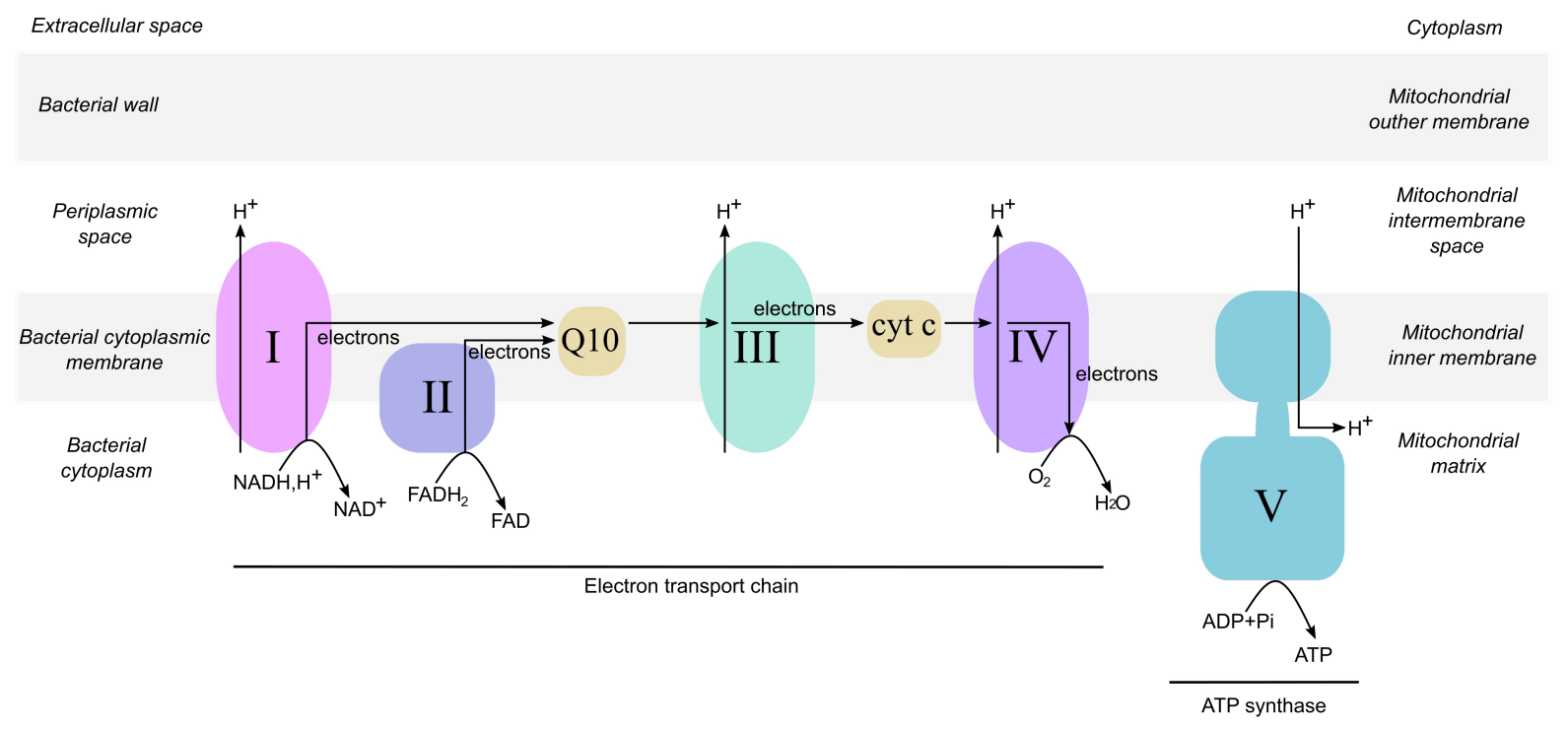

Figure 3. Bacterial and mitochondrial respiratory chain.

In bacteria, the respiratory chain is located in the cytoplasmic membrane (left panel, italics), and in the inner mitochondrial membrane in eukaryotes (right panel, italics). It is composed of an electron transport chain that creates a proton gradient in the periplasmic space for bacteria and in mitochondrial intermembrane space for eukaryotes. Then, this proton gradient is translocated to the bacterial cytoplasm for bacteria or to the mitochondrial matrix for eukaryote through the ATP synthase. This structurally allows the rotation of the ATP synthase, and ATP will be converted from an ADP + Pi reaction.

The electron transport chain is constituted by four large protein complexes (I, II, III and IV) as well as two membrane shuttles, coenzyme Q10 and cytochrome c (Figure 3) [16]. Complex I, also called NADH dehydrogenase, is a complex of approximately $1000 \mathrm{kDa}$ and is composed of 45 protein subunits, 1 flavin mononucleotide coenzyme (FMN, similar to FAD) and 8 iron-sulfur clusters. It catalyzes the transfer of electrons from $\mathrm{NADH}, \mathrm{H}^{+}$to coenzyme Q10, while translocating protons across the inner mitochondrial membrane into the intermembrane space $[17,18]$. Complex II, also named succinate dehydrogenase is a complex of approximately $140 \mathrm{kDa}$ and is composed of 4 protein subunits, 1 flavin adenine dinucleotide coenzyme (FAD), 3 iron-sulfur clusters and 1 single heme group. It catalyzes the transfer of electrons from succinate to coenzyme Q10 [18]. Complex III, named cytochrome c reductase, is a complex of approximately $250 \mathrm{kDa}$ and is composed of 11 subunits, 1 iron-sulfur cluster and 3 cytochromes ( 1 cytochrome $\mathrm{c}_{1}$ and 2 cytochromes b). It catalyzes the transfer of electrons from coenzyme Q10 to cytochrome c, and it translocates protons across the inner mitochondrial membrane into the intermembrane space [18]. Complex IV, also named cytochrome $\mathrm{c}$ oxydase, is a complex of approximately $200 \mathrm{kDa}$ and is composed of 13 subunits, 3 copper atoms and 2 heme groups, also known as cytochrome a and cytochrome аз. It catalyzes the transfer of electrons from cytochrome $\mathrm{c}$ to oxygen (the last acceptor molecule for electrons) and it translocates protons across the inner mitochondrial membrane into the intermembrane space [18]. The shuttles function as follows: coenzyme Q10, also called $\mathrm{COQ}_{10}$ or ubiquinone-10, is a liposoluble electron carrier and transports both electrons and protons [19], while cytochrome $\mathrm{c}$ is a water-soluble protein which carries electrons exclusively [20]. 
In summary, the role of these first 4 complexes is to oxidize electron-donor molecules such as $\mathrm{NADH}, \mathrm{H}^{+}$and $\mathrm{FADH}_{2}$, which are derived from the Krebs cycle, and which are powered by the catabolism of carbohydrates, lipids and proteins. The electrons are then transferred to $\mathrm{O}_{2}$, thereby making this process aerobic. This successive oxidation of donor molecules creates a proton gradient, allowing the phosphorylation of an ADP molecule into ATP by the ATP synthase complex. This process is called phosphorylating oxidation, or more commonly known as oxidative phosphorylation. The ATP synthase is the fifth complex of respiratory chain. We will deepen the description of this ATP factory in the next paragraph [8].

\subsection{ATP synthase}

The process of ATP synthesis by oxidative phosphorylation is a phenomenon already existing in bacteria, that have a membrane respiratory chain very similar to the mitochondrial respiratory one (Figure 3). This similarity between the bacterial and mitochondrial respiratory chain became one of the pillars of the theory of primary endosymbiosis [21-23]. Briefly, this theory explains the origin of mitochondria in eukaryotic cells as the result of the endocytosis of bacteria by a primitive eukaryotic cell. With evolution, it is thought that this primitive eukaryotic cell maintained an endosymbiotic relationship with bacteria, which transformed into current mitochondria approximately between 1.5 to 2 billion years ago [24]. Although this theory is increasingly controversial [25-27], the first studies on ATP synthase were carried out on the bacterial ATP synthase. This is not only due to its similarity with the human ATP synthase, but also because of the fast replication rates of bacteria and the ease of introducing modifications to its genome [28].

ATP synthase is a large complex of about $600 \mathrm{kDa}$ and consists of three parts: the F0 part, the F1 part and a peripheral stalk. The F0 part is embedded in the inner mitochondrial membrane (or the bacterial plasma membrane), and it is responsible of proton translocation (Figure 4). This part rotates within the membrane due to its interaction with protons, hence its nickname "rotor". F0 also forms a central stalk connecting it to the F1 part. The F1 part protrudes in the mitochondrial matrix (or the bacterial cytoplasm) and is the static part of the ATP synthase. F1, also called "stator", is responsible for the phosphorylation of ADP into ATP. In addition to a direct connection between F0 and F1 by the central stalk, the two parts are also connected by a peripheral stalk which further stabilizes the complex and allow to keeps the whole ATP synthase complex stable and static during the rotation of the c-ring and the central stalk within the hexamere $\alpha_{3}-\beta_{3}$ [29] (Figure 4).

From an ultrastructural point of view, F0, F1 and the peripheral stalk are very similar between the bacterial and mitochondrial ATP synthases. On one hand, the bacterial F0 region is composed of subunits a-c9-15, the F1 region is composed of subunits $\alpha_{3}-\beta_{3}-\gamma-\varepsilon$ and the peripheral stalk is composed of subunits $b_{2}-\delta$ [28]. On the other hand, the mitochondrial F0 region is composed of subunits a-cs-15, the F1 region is composed of subunits $\alpha_{3}-\beta_{3}-\gamma-\delta-\varepsilon$ and the peripheral stalk is composed of subunits OSCP-b2-d-F6. In addition to the differences observable between the two peripheral stalks, the mitochondrial ATP synthase is composed of some additional subunits - e, g, f, A6L, j and k - which are associated to the F0 part [30,31] (Figure 4). 

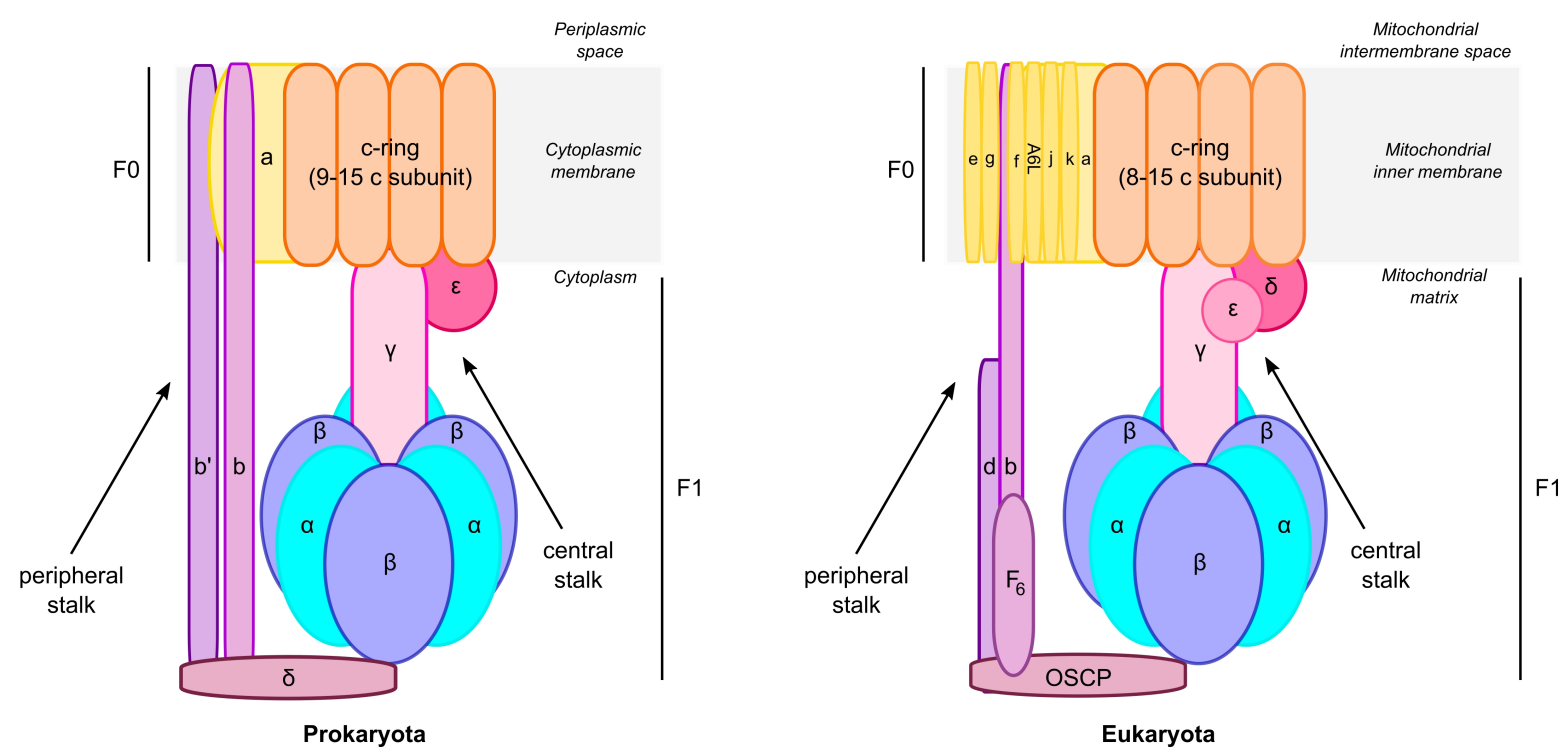

Figure 4. Structural comparison of the bacterial and mitochondrial ATP synthases

Left panel: the bacterial ATP synthase consists of a rotor part F0 (yellow, orange), forming the proton channel by subunit a and c9-15. F0 also carries a central stalk (pink), composed by the $\gamma$ and $\varepsilon$ subunits. The static part F1 is formed by the peripheral stalk (purple) and the catalytic headpiece (blue). The peripheral stalk is constituted by the $\mathrm{b}, \mathrm{b}^{\prime}$ and $\delta$ subunits, and the catalytic headpiece by the $\alpha_{3}-\beta_{3}$ subunits (blue). Right panel: Mitochondrial ATP synthase consists of rotor part F0 (yellow, orange), forming the proton channel by subunit a and c8-15, in addition to subunits e, g, f, A6L, $\mathrm{j}$ and $\mathrm{k}$. As in bacteria, F0 also carries a central stalk (pink) composed by the $\gamma, \delta$ and $\varepsilon$ subunits. The static part F1 is formed by the peripheral stalk (purple) and the catalytic headpiece (blue). The peripheral stalk is constituted by the $\mathrm{b}, \mathrm{d}, \mathrm{F} 6$, OSCP subunits and the catalytic headpiece by the $\alpha_{3}-\beta_{3}$ subunits (blue). Note: The mitochondrial subunit $\delta$ corresponds to the bacterial subunit $\varepsilon$, whereas the mitochondrial $\varepsilon$ subunit does not exist in bacteria.

Being structurally very similar, the bacterial and mitochondrial ATP synthases synthesize ATP on the same principle, the rotational catalysis (Figure 5). As introduced above, the electron transport chain causes an efflux of protons towards the mitochondrial intermembrane space (or bacterial periplasmic space), thereby creating a proton gradient. Through the F0 part of ATP synthase, the flux of protons is then re-internalized from the mitochondrial intermembrane space (or bacterial periplasmic space) to the mitochondrial matrix (or bacterial cytoplasm). Mechanistically, the proton flux induces the rotation of the rotor, thanks to the interactions between protons and aspartate residues carried by the $\mathrm{c}$ subunits of the $\mathrm{F} 0$ region. The rotation of $\mathrm{F} 0$, including the central stalk which is in the core of the F1 region, results in conformational changes of the $\alpha_{3}-\beta_{3}$ hexamer.

The nucleotide-binding sites are located at the interface of $\alpha$ and $\beta$ subunits. It is at the level of the $\beta$ subunits that ATP is directly synthesized, thanks to the presence of a catalytic site within each subunit. The $\alpha$ subunits do not play an active role in catalysis, but they participate to the regulation of the ATP synthase activity [31]. Each of the $\beta$ subunit has a given conformation (Figure 5): the first is the loose conformation, also called $\beta_{\mathrm{DP}}$ because it binds ADP and inorganic phosphate; the second is the tight conformation or $\beta$ TP, because it forms a bond between ADP and Pi and synthesizes ATP. The last is the open conformation, also called $\beta_{\mathrm{E}}$ or empty, because it releases the newly-formed ATP molecule. These different conformations work in synergy to synthesize ATP and at each timepoint, each of the $\beta$ subunits is occupied by a nucleotide [32]. 


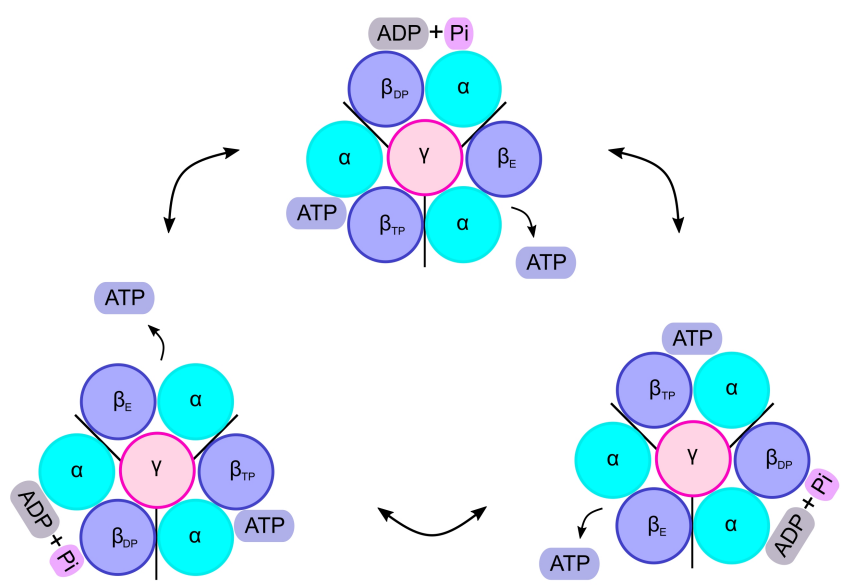

Figure 5. Rotational catalysis is the mechanism of ATP production by the ATP synthase

Each of the $\beta$ subunits of the ATP synthase are found in a loose, tight or open conformation allowing the synthesis and the release of ATP molecules. The loose conformation $(\beta \mathrm{DP})$, corresponds to the binding of ADP and inorganic phosphate. The tight conformation $\left(\beta_{\mathrm{TP}}\right)$, corresponds to the formation of a bond between the ADP and inorganic phosphate. The open conformation $(\beta \mathrm{E})$, corresponds to the release of the newly-formed ATP molecule into the mitochondrial matrix (or bacterial cytoplasm).

A peculiarity of the mitochondrial ATP synthase is that it can arrange in supramolecular complexes as dimers and oligomers. The clustering of multiple ATP synthase complexes triggers local folding events on the inner mitochondrial membrane, commonly known as mitochondrial cristae $[33,34]$.

Overall, ATP production is a major and complex cellular process. Given that the intracellular functions of this molecule are critical for cell physiology and in pathological conditions, monitoring the levels of ATP in cells has been used for decades as readout of cellular functions. First analyzed using biophysical and biochemical approaches, the raise in microscopy-based techniques has deepened our understanding of the many roles of ATP in the cell. In the next chapter, we will make an overview of the various approaches currently available to study ATP.

\section{Techniques to monitor ATP levels}

There are several approaches to estimate the levels of ATP, ADP and AMP (Adenosine monophosphate). In the section below, we will first describe the most employed biochemical and biophysical assays to measure cellular and extracellular ATP levels. Second, we will review the panel of microscopy-based tools and approaches to monitor cellular and extracellular ATP levels at the single-cell level and with spatiotemporal resolution.

\subsection{Biochemical and biophysical approaches to measure cellular and extracellular ATP levels}

\subsubsection{Nuclear Magnetic Resonance spectroscopy}

Nuclear Magnetic Resonance (NMR) is a technique allowing to determine the structure of molecules, and largely employed to unravel the conformation of macromolecules. This technique is based on the property of specific atoms to have an odd number of protons and/or neutrons. This results in a non-zero nuclear spin (I) for atoms as ${ }^{1} \mathrm{H},{ }^{13} \mathrm{C},{ }^{19} \mathrm{~F}$ and ${ }^{31} \mathrm{P}$, which have an I value equal to $1 / 2$ making them comparable to magnetic dipoles. When these atoms are subjected to a magnetic field, they absorb an electromagnetic wave and resonate by emitting an energy peak detectable with a NMR spectrometer [35]. Since ATP, ADP and AMP molecules contain a significant proportion of $\mathrm{H}$ and P atoms, it is possible to detect them using this approach [36-38] (Figure 6). 


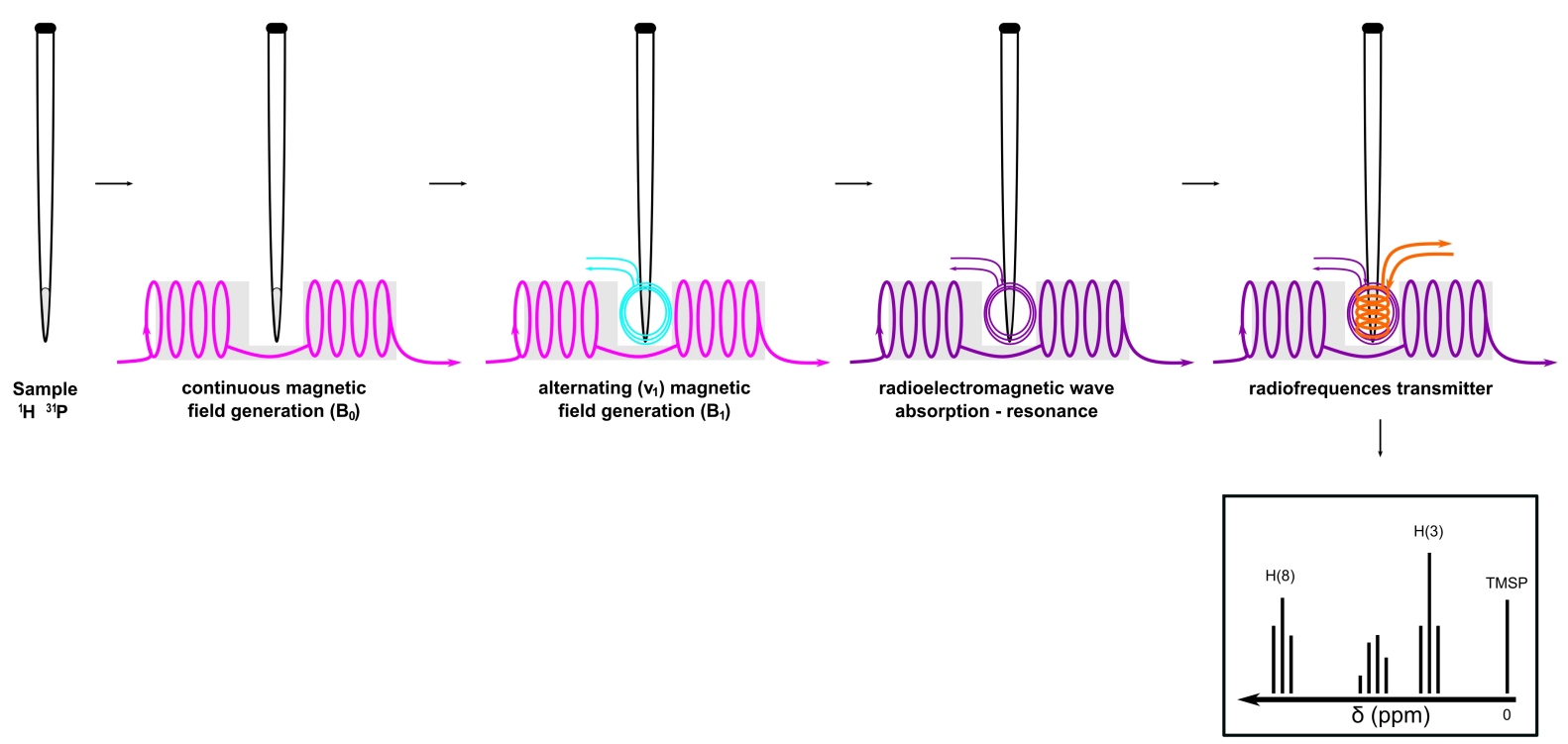

Figure 6. Principle of Nuclear Magnetic Resonance

The spectrometer consists of a magnet and several coil systems. The sample is placed in a superconducting magnet (or electromagnet), in which a continuous magnetic current (magenta) is generated, creating a $\mathrm{B}_{0}$ magnetic field of up to 25 Tesla [39]. Then, a radiofrequency oscillator generates an alternating magnetic current (cyan) $\mathrm{B}_{1}$ at the frequency $v_{1}$, called resonance frequency, of 60 to $950 \mathrm{MHz}$ [40,41]. A radio-electromagnetic wave is thus generated (purple). The studied atom absorb this radio-electromagnetic wave, and its capable to resonate. It will then emit energy peaks that can be captured on an NMR spectrum thanks to a radiofrequences transmitter (orange), which will detect and recover the radiofrequencies and transmits them to computer software. On this spectrum, the reference (peak at $0 \mathrm{ppm}$ on the x-axis) corresponds to the internal standard, which is a molecule with a known resonance frequency and that can be used as a reference. This reference compound (usually TMSP) has very low toxicity, an overall chemical inertia and emits a single peak on the spectrum make it readily distinguishable from all other molecules tested. Since ATP, ADP and AMP molecules contain a significant proportion of $\mathrm{H}$ and $\mathrm{P}$ atoms, their relative signals on the NMR spectrometer will differ from the one of the internal standard.

The quantitative Nuclear Magnetic Resonance spectroscopy (qNMR) is a variant of NMR allowing, as the name indicates it, to quantitatively measure one or more molecules simultaneously [42]. On a qNMR spectrum, the peak area is directly proportional to the number of atoms, and the concentration of the molecules analyzed can be determined by using an internal standard of known concentration previously added to the sample. By using ${ }^{1} \mathrm{H}$ and ${ }^{31} \mathrm{P}$ qNMR ( ${ }^{1} \mathrm{H}-\mathrm{NMR}$ and ${ }^{31} \mathrm{P}-\mathrm{NMR}$ ), Lian et al. were able to simultaneously monitor ATP, ADP and AMP [38]. As internal standards, they employed TMSP (sodium 3-(trimethylsilyl) propionate-2,2,3,3-d4) for ${ }^{1} \mathrm{H}-\mathrm{NMR}$, and $\mathrm{Na}_{2} \mathrm{HPO}_{4}$ (anhydrous disodium hydrogen phosphate) for ${ }^{31} \mathrm{P}-\mathrm{NMR}$. The main advantage of this method is the perfect linearity in a given range: $0.1-100 \mathrm{mM}$ for ${ }^{1} \mathrm{H}-\mathrm{NMR}$, and $1-75 \mathrm{mM}$ for ${ }^{31} \mathrm{P}-\mathrm{NMR}$. qNMR turned out to be particularly useful in pathological situations where cytoplasmic ATP is massively released into the circulation [43]. However, this technique has some limitations: first, the detection range is lower than the concentration of physiological ATP present in cells and tissues. For example, ATP concentration in human plasma is around $0,001 \mathrm{mM}$ [44]. Moreover, this technique has a low temporal resolution, as requires an extended time frame - approximately of 14 hours - to complete the detection of a specific spectrum. This long acquisition time is a significant caveat for specific applications as high-throughput screening. In addition, and although extremely precise in estimating the quantity of specific components, qNMR requires significant amounts of biological materials (e.g. total cell extracts) to provide reliable readouts on the concentration of nucleotides in physiological conditions. Lastly, NMR requires very expensive equipments that prevent its thorough diffusion in every lab interested in estimating ATP levels. 


\subsubsection{High-Performance Liquid Chromatography and Liquid Chromatography coupled to tandem Mass Spectrometry}

High-Performance Liquid Chromatography (HPLC) is a widespread technique, which has been widely used since the 1990s. Its purpose is to separate molecules within a complex sample according to specific features as polarity [45]. This method is based on a mobile phase, which is constituted by the sample to analyse, previously resuspended in a solvent with a given polarity. The mobile phase then passes through a column, which constitutes the stationary phase. The molecules of the sample will be separated thanks to the different affinities that they have with the column. Those with more affinity will be retained on the stationary phase, while the others will be washed out. As a result, the molecules will be separated and will exit the column at different times. At the exit of the stationary phase, the molecules will pass through a detector for analysis, thus generating a chromatogram (Figure 7). Globally, this approach allows to identify the molecules and their absolute quantity [45].

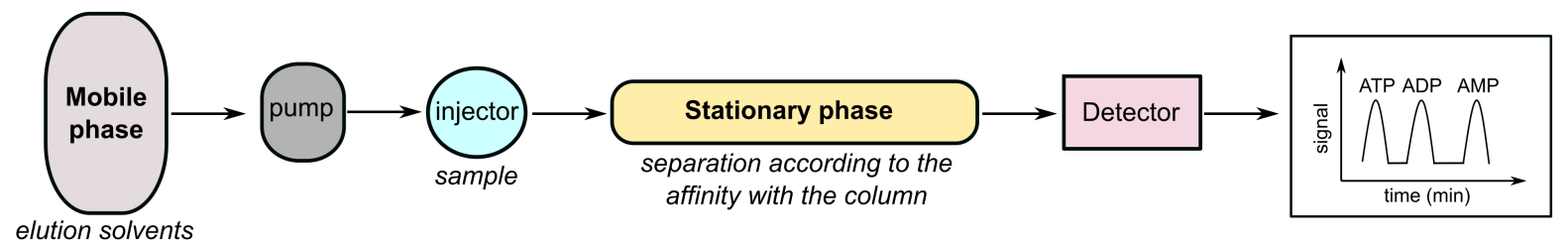

Figure 7. High-Performance Liquid Chromatography Principle

The mobile phase, constituted by solvents of more or less polar, will pass through a pump which will put the mobile phase under pressure. Then, the injector will put the samples in contact with the mobile phase, which then passes through the stationary phase. The stationary phase is a column that will separate the different molecules of the sample according to their affinity the solvents. At the exit of the column, the detector will detect the molecules of interest by a signal (different types of detectors exist as UV-visible radiation, fluorescence emission, mass spectrometer). The information will then be processed by a computer, which will then generate a chromatogram as output.

HPLC has thoroughly been used to determine ATP, ADP and AMP levels in different types of samples: in plant primary cells [46], in conventional mammalian cell lines such as MCF7 and MDA436 [47], or in primary cells such as human and mouse platelets [48]. The sensitivity of HPLC in detecting nucleotides is in the order of $\mu \mathrm{mol}$, therefore HPLC analyses on biological samples require large amounts of cells and a complete extraction of cell contents. This is a potential caveat, as complete extractions can lead to the loss of nucleotides due to hydrolysis. To counteract this limitation, Bhatt et al. have developed an ion-pairing HPLC method coupled with fluorescence detection to quantify ATP, ADP and AMP in primary astrocytes [49]. In ion-pairing HPLC, the stationary phase consists of an ion-pairing column, and an ion pair reagent is added to the mobile phase to increase or decrease the retention time of partly-ionized organic analytes in the column, according to the type of molecule to analyse. In addition, the method to detect nucleotides at the exit of the column benefits from the sensitivity of fluorescence ( $\mathrm{nmol} / \mathrm{pmol}$ range). Nucleotides undergo a derivatization by the transformation of adenine-based nucleotides into 1, $\mathrm{N}^{6}$-ethenodericatives, which gives them fluorescent-like properties and protects them from hydrolysis during cell extraction. This method shows an excellent selectivity thanks to the use of an excitation wavelength at $280 \mathrm{~nm}$, and by recording the emission at $410 \mathrm{~nm}$, which is specific for etheno-adenine compounds. Unlike conventional HPLC, ion-pairing HPLC is extremely precise and capable to monitor the levels of nucleotides at the pmol scale. However, it still requires complete cell extraction and a modification step to make ATP, ADP and AMP fluorescent. Compared to NMR, HPLC is a fast (the retention time is about 20min [49]), automated, highly reproducible and very accurate method to identify nucleotides in total cellular extracts. As for NMR, this technique is very expensive, thereby hindering its diffusion as a gold-standard method to quantify nucleotides in living samples. 
The Liquid Chromatography coupled to tandem Mass Spectrometry (LC-MS/MS) is a combination of the ability to separate different molecules by liquid chromatography as in HPLC, and the ability to detect and identify molecules of interest by measuring their mass using mass spectrometry. Briefly, mass spectrometry is based on the ionization of molecules of interest (generally by ElectroSpray Ionization, ESI; or Matrix Assisted Laser Desorption Ionization, MALDI) and their sublimation (passage of molecules to the gas state) [50]. Once ionized, the molecules enter an acceleration zone in which they acquire a specific speed, which directly depends on their mass $(\mathrm{m})$ and charge ( $\mathrm{z}$ ). Then, the molecules are sorted according to their $\mathrm{m} / \mathrm{z}$ ratios in an analyzer, which is constituted by an empty column of air preserving the respective speed of each molecule against friction forces. Once the molecules have gone through the analyzer, an ion detector detects the molecules one by one and generates a spectrum (Figure 8).

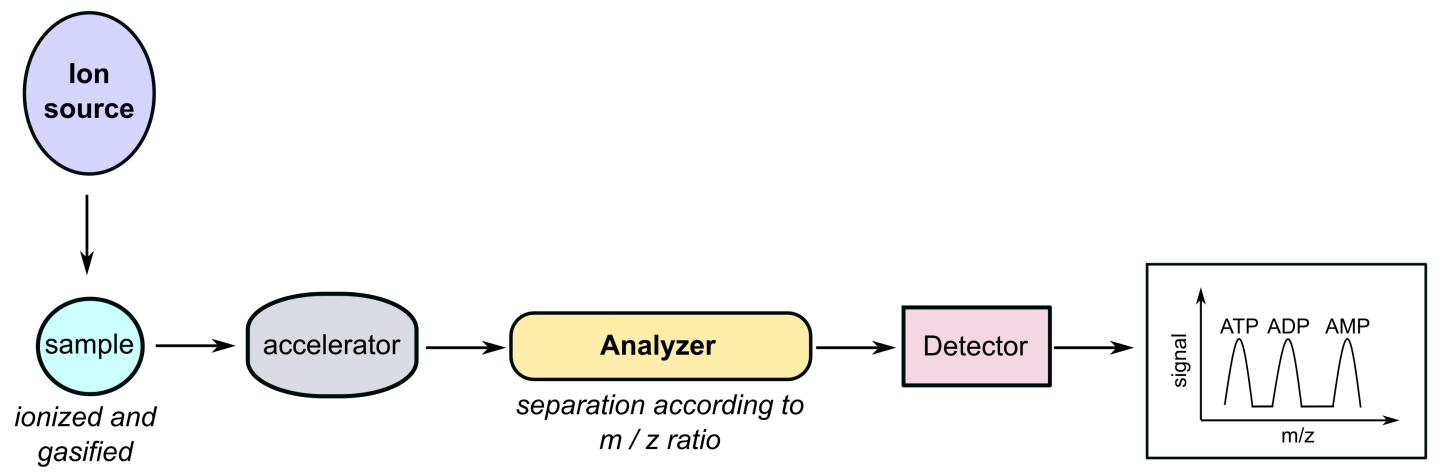

\section{Figure 8. Principle of Mass Spectrometry}

First of all, the sample is prepared by integrating it into a matrix, then put in a specific plate and placed in the spectrometer. The ion source (nitrogen laser) irradiates the sample (the matrix protects the sample from destruction by direct laser irradiation). This irradiation causes heating, which transforms the sample from a solid state to a gaseous state. Then, this irradiation causes shocks between the molecules of the sample and the matrix, which will cause the transfer of protons from the matrix to the sample, thus leading to ionization. The sample is therefore gasified and ionized (positively charged). Furthermore, the sample passes through an acceleration zone which is negatively charged. The molecules of the sample therefore acquire a certain speed, which is inversely proportional to the mass $(\mathrm{m})$ or charge $(\mathrm{z})$ of the molecule. Then, the molecules enter the analyzer which will separate the molecules according to their $\mathrm{m} / \mathrm{z}$ ratio. At the exit of the analyzer, the molecules of interest will be detected and processed by a computer to generate a spectrum.

The LC-MS/MS method benefits from the advantages of both techniques: LC allows to separate molecules according to their polarity, while MS/MS allows to determine their quantity with high precision. Recent studies used LC-MS/MS for the simultaneous quantification of ATP and other small metabolites as 2,3-diphosphoglycerate, $\mathrm{NAD}^{+} / \mathrm{NADH}, \mathrm{H}^{+}$or short chain acyl-CoAs [51,52]. Therefore, this method makes it possible to obtain the concentration of ATP and that of other molecules with very different biochemical properties, at the same time and faster than the previous approaches (retention time is between 10 min [51] and 2min [52], according to the setup used). However, like HPLC, LC-MS/MS is an extremely expensive technique as it requires expensive instruments and sample preparation can be cumbersome.

\subsubsection{Respirometry}

Respirometry (or oxygraphy) is an indirect method to measure the mitochondrial ATP production. As described above, the electron transport chain creates a proton gradient by consuming oxygen, which in turn allows the ATP synthase to rotate and produce ATP from ADP + Pi (Figure 3). Oxygen consumption can directly be linked to ATP production: the more cells produce ATP, the more oxygen they consume [53]. The principle of oxygraphy is to modulate the respiratory chain using 
selective drugs known for their capacity to activate or inhibit its activity, and to measure the corresponding flux of oxygen. Concretely, respirometry consists of placing cells (intact or permeabilized) or mitochondrial fractions in oxygen chambers known as Clark's electrodes. The drugs are then injected sequentially into the chambers, while the electrodes will measure the oxygen levels after the injection of each drug. The cocktail of drugs used in an oxygraphy experiment normally contains: oligomycin, FCCP (carbonylcyanide p-trifluoromethoxyphenylhydrazone) and a mix of antimycin-rotenone (Figure 9).
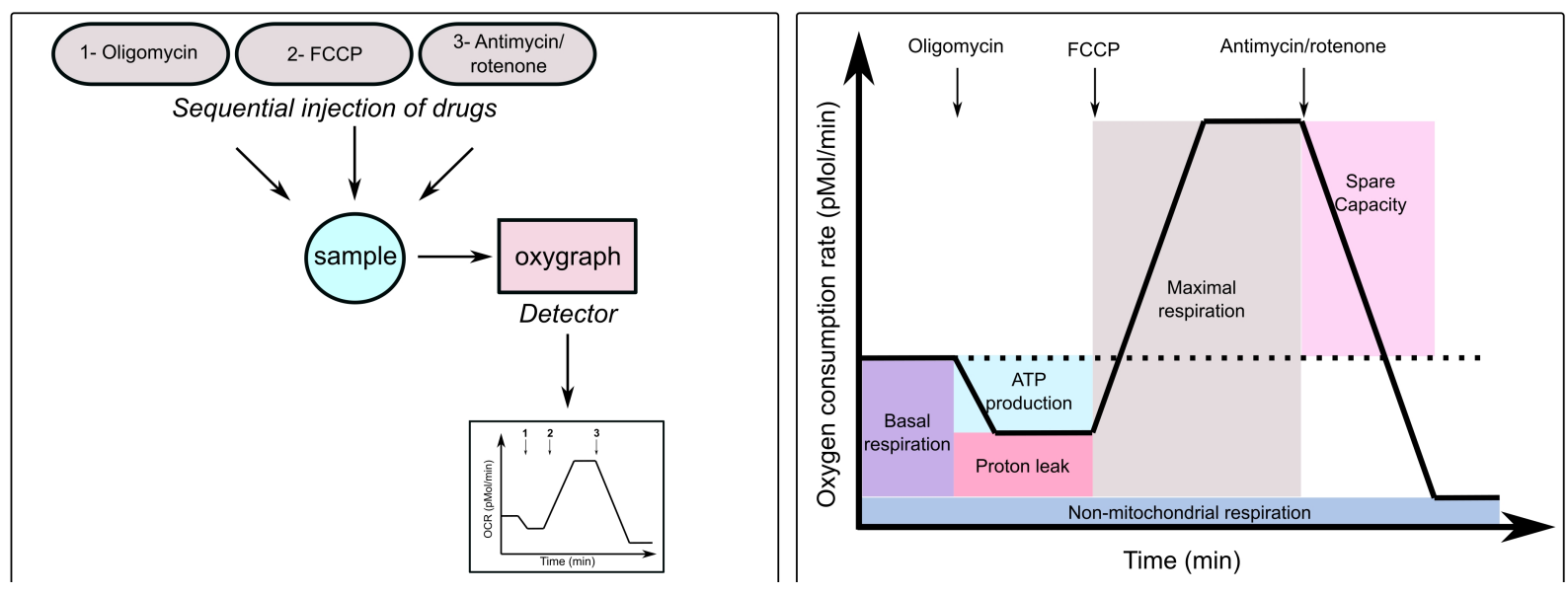

Figure 9. Respirometry: principle, drugs used and expected output

Left panel: The oxygraph is equipped with sensors, called Clark's electrodes, which measure oxygen consumption of the sample in each chamber. The reduction of $\mathrm{O}_{2}$ to $\mathrm{H}_{2} \mathrm{O}$ produces an electrical current which is amplified by the device. The intensity of the current is proportional to the amount of dissolved $\mathrm{O}_{2}$ in the measured chamber. Moreover, the sequential injection of drugs which acts on mitochondrial respiratory chain allows to dermine the metabolic profil by oxygen consumption rate of cells or mitochondrial isolates. Right panel : The oxygen consumption rate varies depending on the drugs injected on a cell type or mitochondrial isolates. First without drug, we determine the basal respiration. Then, by adding oligomycine we determine the proportion of ATP production by ATP synthase. Then by adding FCCP, we determine the maximum respiratory capacity in other words the maximal capacity to reduce oxygen. Finally by adding antimycin/rotenone mix, we determine the spare capacity in other words the maximal energy production capacity [54].

Oligomycin is an antibiotic which inhibits the ATP synthase by associating with the F0 subunit, and prevents the backflow of protons to the mitochondrial matrix [55]. This results in low or no oxygen consumption, and thus no ATP production. FCCP is a weak and lipophilic acid which acts as an ionophore and a decoupling agent. Its lipophilic properties allow FCCP protonation in the intermembrane space. Then, FCCP crosses freely the inner mitochondrial membrane and returns to the mitochondrial matrix. Once there, it deprotonates itself before re-crossing freely the inner mitochondrial membrane and being re-protonated in the intermembrane space. Because of these protonation/deprotonation cycles on the FCCP molecule, the protons bypass the ATP synthase almost completely. Oxygen consumption is therefore maximized, but there is almost no ATP synthesis by the ATP synthase [56]. Last, antimycin is an antibiotic blocking the electron transfer at complex III (Cytochrome c reductase) [57]. It is often used in combination with rotenone, an insecticide blocking electron transfer at complex I (NADH deshydrogenase) [58]. The antimycin/rotenone mix blocks the overall electron transfer of the respiratory chain and as a consequence, the electrons can not be transferred to oxygen. As a result, the proton gradient no longer exists, and ATP synthesis by the ATP synthase stops (Figure 10). Respirometry was shown to be a convenient and less expensive technique than NMR and HPLC to measure oxygen consumption. It allows to infer on ATP production in a wide variety of cells and in mitochondrial homogenates. It also provides information on mitochondrial quality, in terms of ATP production levels and on the activity of respiratory chain complexes. 


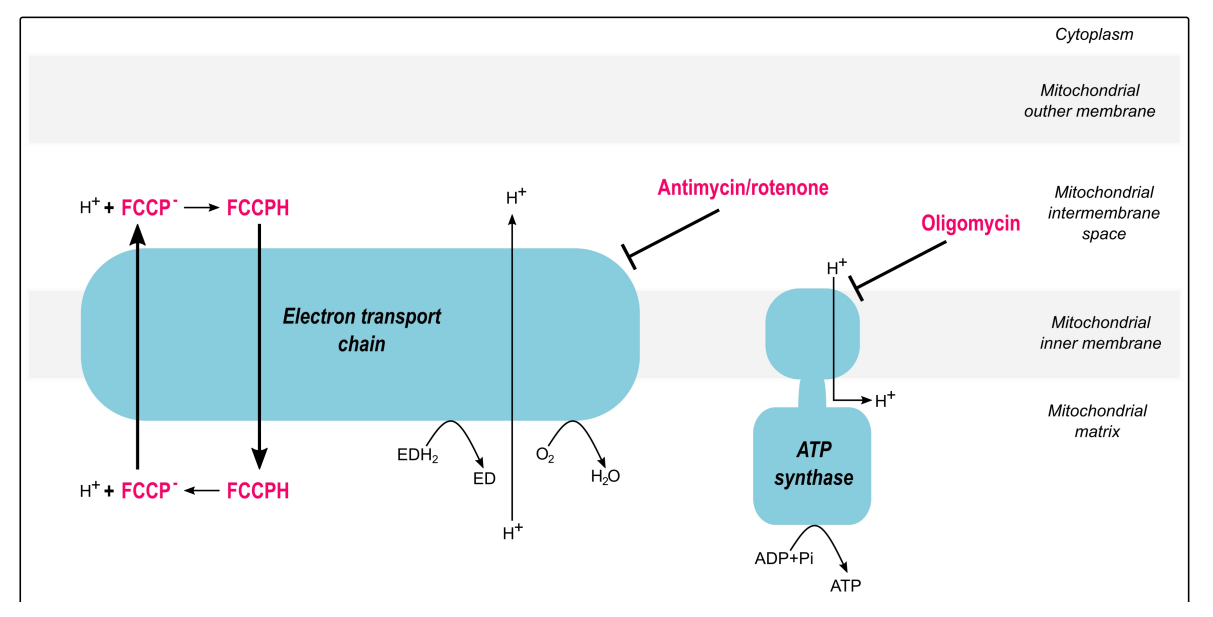

Figure 10. Representation of the mechanisms of actions of the drugs used in respirometry.

Oligomycin inhibits ATP production by blocking proton influx on the ATP synthase. By associating with complex III, antimycin inhibits ATP production by inhibiting proton gradient formation. Also, by associating with complex I, rotenone inhibits ATP production by inhibiting proton gradient formation. FCCP inhibits ATP production by inhibiting proton reflux through ATP synthase. ED=Electron Donor molecule

In conclusion, NMR, HPLC and LC-MS/MS allow the direct quantification of global ATP levels from cell extracts and serum samples previously denaturated. Conversely, oxygraphy allows to work on intact samples without prior denaturation, but the measurement of ATP production is inferred from oxygen levels, and remains therefore indirect. Overall, these are widely-employed, robust and reproducible methods. However, theses approaches are hardly compatible with real-time analyses, and do not provide a sufficient spatiotemporal resolution. Indeed, phosphorylation of ADP to ATP occurs in seconds, while any analysis performed with the methods described above occurs in minutes or hours [59]. This is why the arrival of tools based on microscopy is a significant advance in the field: as described below, they allow to detect different pools of ATP (i.e. organellar, cellular and extracellular) with high spatiotemporal resolution. Importantly, they improved our understanding of the mechanisms of ATP production at the single-cell level.

\subsection{Microscope-based tools to measure ATP levels}

In the last years, huge efforts have been made to develop tools capable of detecting ATP levels in living cells, but also at the single-cell scale. Microscope-based tools appear to be a convenient way to study ATP in living and single cells with sufficient spatiotemporal resolution. These microscopebased tools are based on their ability to emit light, in other words luminescence. Luminescence is the phenomenon of light emission (therefore photons) from a molecule in an electronically excited state. Indeed when a molecule is excited, it stores energy, which makes it electronically unstable. In order to regain its electronic stability, the molecule returns to its fundamental state by emitting this excitation energy in the form of photons. According the way of molecule-excitation, there are several types of luminescence : electroluminescence, which is the excitation by an electric field; chemiluminescence, which is the excitation by a chemical reaction; bioluminescence, which is the excitation by an enzymatic reaction and can be considered a sub-type of chemiluminescence; and fluorescence, which is the excitation by photon absorption.

In the sections below, we will cover probes responding to the cited principles and we will particularly focus on fluorescent-based biosensors (Figure 11). As a reminder, a biosensor is a tool it consists of a biological molecule - bio - and makes it possible to sense a given activity - sensor - .

Fluorescent-based biosensors are built on three selective properties of fluorophores: 1- the quantum yield of each fluorophore, that is the ratio between the number of photons emitted and the number of photons absorbed; 2- specific excitation and emission wavelengths $(\lambda)$ that allow to use probes 
individually or in combination; 3- the specific lifetime of their excited state that is duration between the excitation of the fluorophore and its return to the fundamental state [60,61] (Figure 11).

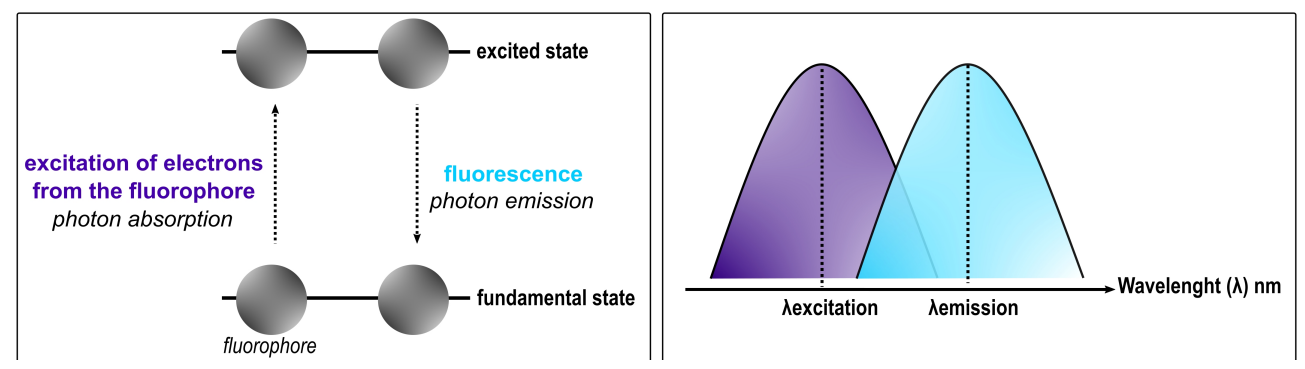

Figure 11. Principle of fluorescence and excitation/emission spectra of fluorophores

Fluorescence is the phenomenon of photon emission by a fluorophore. Left panel: a fluorophore is excited by a laser, it absorbs photons and changes from a stable (or fundamental) state to an unstable (or excited) state. In order to return at the fundamental state, the fluorophore emits photons, thus fluorescence. Right panel: each fluorophore is excitable and emits at a particular wavelength ( $\lambda$ excitation $/ \lambda$ emission, respectively). This constitutes the excitation/emission spectra of the fluorophore.

\subsubsection{Electrochemiluminescence-based tools}

The electrochemiluminescence (ECL) is a technique which is initiated by a step of electron transfer to the surface of an electrode. Several studies use this highly-performing and sensitive technique to detect and quantify ATP [62-64]. Liu et al. developed an ECL ATP sensor which turns On or Off when ATP is bound or unbound, respectively [62] (Figure 12). This method is based on the use of Quantum dots (QDs), aptamers and DNAzyme. QDs, also called "artificial atoms", are semiconductor nanostructures capable of collecting electrical charges and emitting light according to their size. The QDs are attached to an electrode (Glassy Carbon Electrode, GCE), and then linked to a DNA sequence (hereby called DNA1). The DNA1 is partially complementary with the aptamer, a synthetic oligonucleotide capable of binding a specific ligand, as the ATP. The DNA1 allows the aptamer to be linked to the modified electrode of the QDs. The complex "QDs modified electrode DNA1 - Aptamer" forms an aptasensor and will work synergistically with the signal probe called DNAzyme, an oligonucleotide capable of performing a specific chemical reaction. Here, the DNAzyme is carried by a gold nanoparticle and consists of a second DNA sequence (hereby called DNA2), which is also partially complementary to the aptamer and associated with a hemin molecule, a porphyrin playing the role of cofactor by binding electrons. This DNA2-hemin association then forms a secondary structure called G-quadruplex, and it constitutes the signal probe. If there is no ATP, the aptasensor is linked to the signal probe, which is able to reduce oxygen to hydroxide anion and induces a drastic decrease in the ECL emission. Conversely, the aptamer preferentially binds to the ATP molecule when it is present. As a result, the aptamer and the signal probe detach from the QD-modified electrode, allowing a significant ECL emission $\left(\lambda_{\mathrm{em}}=610\right)$. 

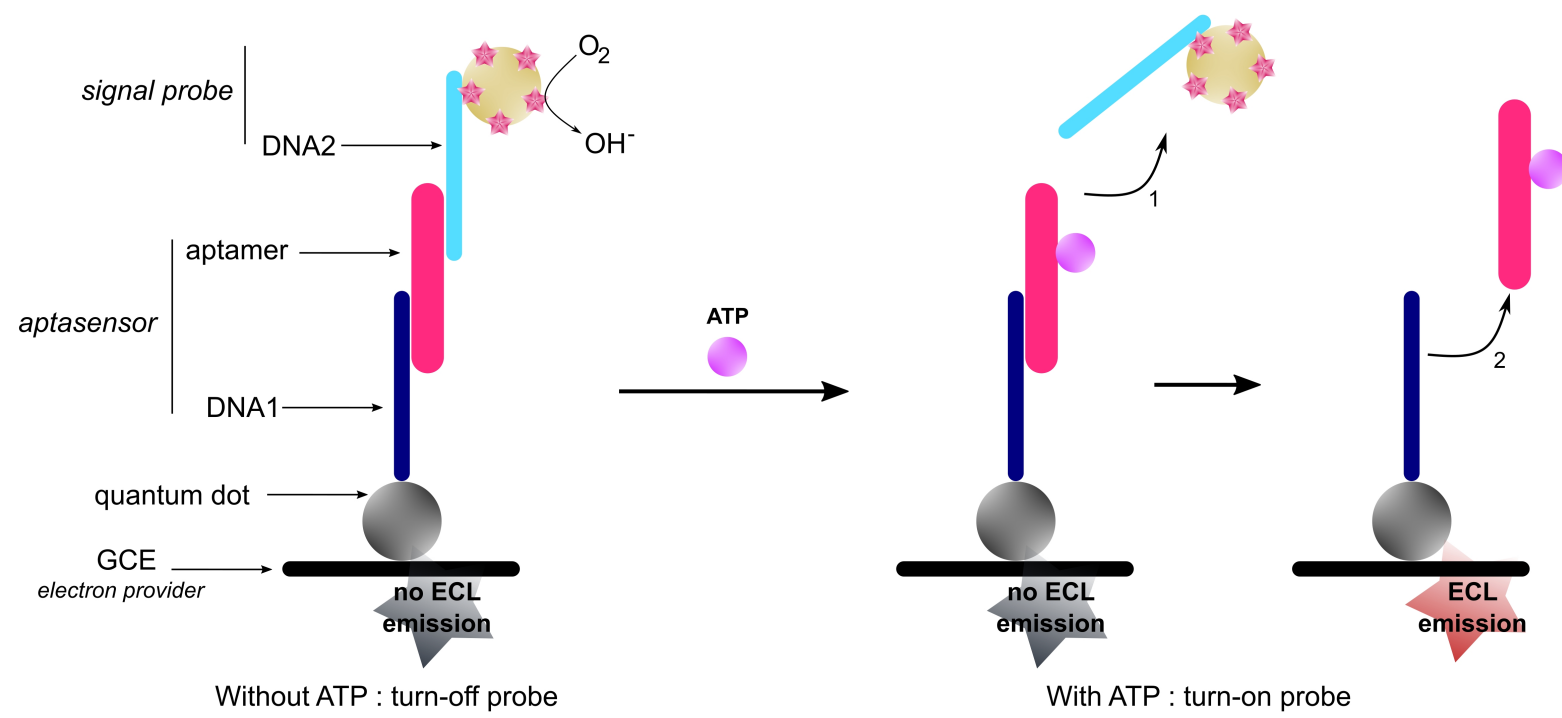

Figure 12. Mode of functioning of an electrochemiluminescence-based ATP probe

This type of luminescence is initiated by electron transfer. The quantum dot is fixed to an electrode (GCE) and then linked to the aptasensor. The aptasensor consists of a DNA sequence (DNA1) and a synthetic oligonucleotide (aptamer) capable of binding ATP. The DNA1 is partially complementary with the aptamer, a synthetic oligonucleotide capable of binding a specific ligand, as the ATP. The aptamer is capable to bind the signal probe. The signal probe is a DNAzyme, which consists of a second DNA sequence (DNA2, also partially complementary with the aptamer) and gold nanoparticle associated with a hemin molecule (cofactors of $\mathrm{O}_{2}$ reduction) Left panel: when there is no ATP, the aptasensor is linked to the signal probe, which is able to reduce $\mathrm{O}_{2}$ into $\mathrm{OH}^{-}$and leading to a drastic decrease in the emission of ECL. Right panel: when there is ATP, the aptamer preferentially binds ATP, which detaches the signal probe and the aptamer and allows a significant ECL emission.

This method of detecting ATP was originally validated on human serum samples, as firstgeneration QDs necessitated a liquid medium for better results. Recently, the use of QDs has become more diffused and although their use in medicine is still largely unexplored, in vivo analyses using mouse models seems promising $[65,66]$. This ECL-based technique seems to be very sensitive to detect ATP levels (detection at nanomolar scale), and the use of quantum dots for live cell imaging is more and more widespread [67]. This technique should be further optimized to use it in single-cell imaging applications and to obtain a better spatiotemporal resolution in living samples.

\subsubsection{Chemoluminescent-based tools}

Chemoluminescent-based tools - chemosensors -, are based on the chemical properties of ATP itself, which is capable of carrying out interactions of different nature (covalent, electrostatic, hydrogen bonds, etc...) with its substrates. This is due to the chemical structure of ATP, containing three negatively charged phosphate group, an aromatic adenosine and a molecule of ribose with several hydroxyl group. The vast majority of chemosensors are single-wavelength ratiometric sensors, therefore requiring a spectrometer to quantify the presence of ATP [61].

The most significant chemosensors are based on rhodamine, a small organic molecule which emits fluorescence due to its xanthene nucleus $\left(\lambda_{\mathrm{em}}=558\right)$. The rhodamine-based probe called Rh6G-NHPBA consists of rhodamine 6G (Rh6G), a diethylenetriamine group $(\mathrm{NH})$ and a phenyl boronic acid group (PBA) (Figure 13). In the absence of ATP, the probe is not fluorescent thanks to its closed ring structure. Conversely, in the presence of ATP three specific interactions will take place. The three interaction are: 1) covalent interactions between PBA and the ribose molecule of ATP, 2) $\pi-\pi$ interactions between the xanthene nucleus of Rh6G and the adenine of ATP, 3) electrostatic interactions between the amino groups of $\mathrm{NH}$ and the phosphate group from ATP [68]. These 
interactions induce the ring to open and free the fluorescent properties of Rh6G, which can then be detected.

The use of small organic molecules allows to obtain an excellent selectivity for ATP over ADP and AMP, but also to monitor mitochondrial ATP fluctuations in live cells [61]. However, some biophysical properties of this probe remain to be elucidated. It has been previously shown that rhodamine B, which is a lipophilic cation, accumulates in the mitochondrial matrix [69]. However, it has been suggested that this accumulation may result from a combination of the negative mitochondrial membrane potential, the high viscosity of the organelle, and ATP concentration in mitochondria [70]. Although rhodamine-based probe turned out to be a real advance for the monitoring of mitochondrial functions and in particular to determinate ATP levels [71,72], they also show very important limitations for live cell imaging, as aggregation, quenching and low photosensitivity [73].
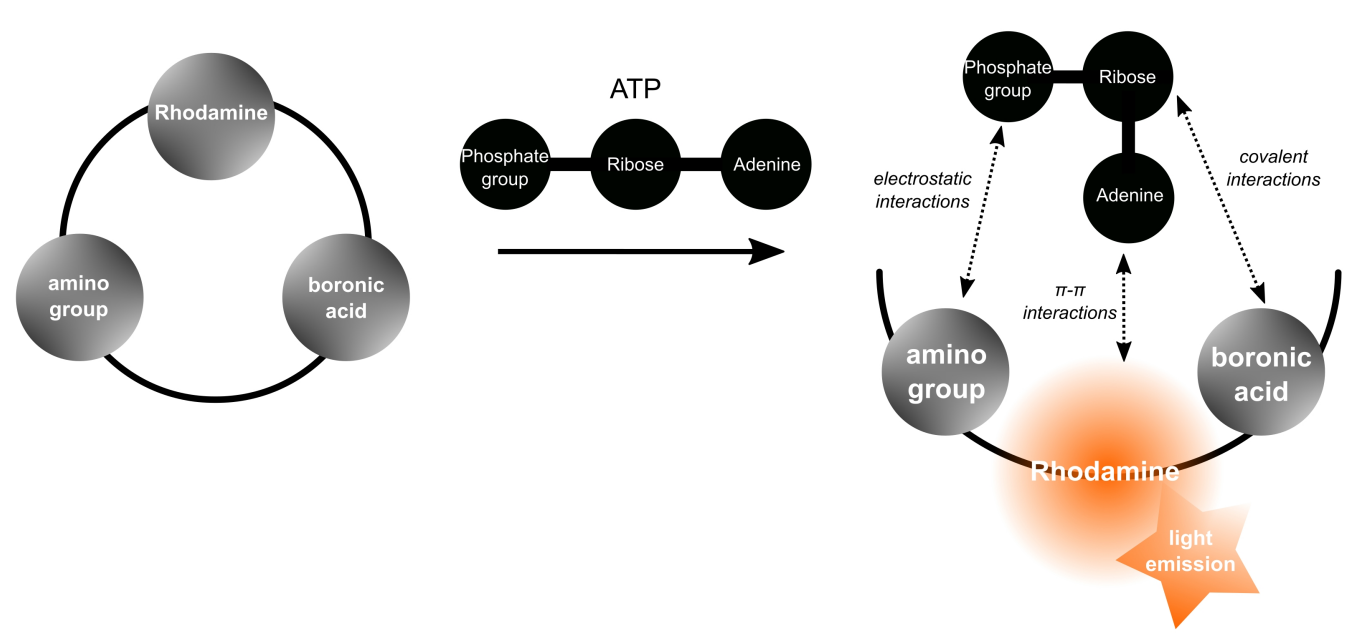

Figure 13. Rhodamine-based ATP reporter

In the absence of ATP, the probe is not fluorescent thanks to its closed ring structure. In the presence of ATP, three specific interactions take place: covalent interactions between PBA and ribose; $\pi-\pi$ interactions between xanthene nucleus of rhodamine and adenine, electrostatic interactions between amino groups and phosphate group. These interactions open the ring and allow the fluorescence of rhodamine to become visible.

\subsubsection{Bioluminescence and BRET-based tools to measure cellular and extracellular ATP levels}

In bioluminescence, the emitted light is produced by an enzymatic reaction. This enzyme is typically a luciferase and in the presence of oxygen, ATP and $\mathrm{Mg}^{2+}$, luciferase oxidises luciferin (its substrate) into oxyluciferin (Figure 14). This oxidation causes the emission of photons, and the resulting emission spectra is generally comprised between $546 \mathrm{~nm}$ and $618 \mathrm{~nm}$ according to the luciferase type [74]. Therefore, the capacity of luciferin to emit light is linked to the available amount of ATP in the surrounding environment. Light emission can thus be used to determine the concentration of ATP in plasma, mitochondria isolates or tissue extracts (global extraction of nucleotides can be performed by using perchloric acid) [75,76]. By using this luciferase-based enzymatic system, it is possible to create a localisation-specific luciferase by targeting cell compartments (mitochondria, cell membrane, nucleus...) by adding specific localisation signal to the luciferase [77].

Rangajaraju et al. have developed a luciferase-based enzymatic system capable to monitor presynaptic ATP concentration [78]. This luciferase-based tool is called Syn-ATP, and it consists of synaptophysin (major synaptic vesicle protein) fused with Luciferase and mCherry. The use of synaptophysin allows the targeting of the probe to nerve terminals, and the use of mCherry helps to determine the total amount of luciferase by performing a luminescence / fluorescence ratio. The SynATP biosensor is a useful tool for researchers interested in exploring synaptic functions: the more ATP is consumed, the more bioluminescence levels - and therefore synaptic activity - are significant. 
These types of solutions for ATP quantification are widely used and commercialised by several companies. However, this method has some notable caveats. First, certain luciferases have the capacity to produce ATP from the pool of ADP present in the sample, thereby showing poor specificity for the ATP produced after the oxidation of luciferin [79]. In addition, the enzymatic activity of luciferases can be perturbed by cellular inhibitors and by others cellular activities which may reduce their capacity to perform bioluminescence [80].

$$
\text { Luciferin }+\mathrm{ATP}+\mathrm{O}_{2} \overbrace{}^{\text {Luciferase }} \text { Oxyluciferin }+\mathrm{AMP}+\mathrm{PPi}+\mathrm{CO}_{2}+\text { Light }
$$

Figure 14. Principle of the luciferase reaction in luciferase-based ATP assays.

In presence of luciferin, oxygen and ATP, the luciferase generate oxyluciferin, AMP, pyrophosphate anion, $\mathrm{CO}_{2}$ and photons emission (light)

In order to overcome these problems, the bioluminescence property of luciferase-like enzymes is used to develop biosensors based on the BRET (Bioluminescence Resonance Energy Transfer) phenomenon. BRET is based on the energy transfer between a bioluminescent molecule as luciferase, to a fluorophore. In the case where the luciferase and the fluorophore are close - less than $10 \mathrm{~nm}$ apart - the energy derived from a luciferase reaction excites the fluorophore [81]. BRET can be classified as intermolecular BRET and used to study the interactions between two different proteins or protein oligomerization. If it is used to study the conformational changes of a protein, it will be classified as intramolecular BRET. BRET-based biosensors rely on intramolecular BRET to monitor ATP (Figure 15). These tools rely on a luciferase that does not require the consumption of ATP to oxidize luciferin into oxyluciferin (Figure 14). Therefore, these non-consuming ATP luciferases do not consume endogenous ATP and thus do not disturb the sensing of ATP in biological system.

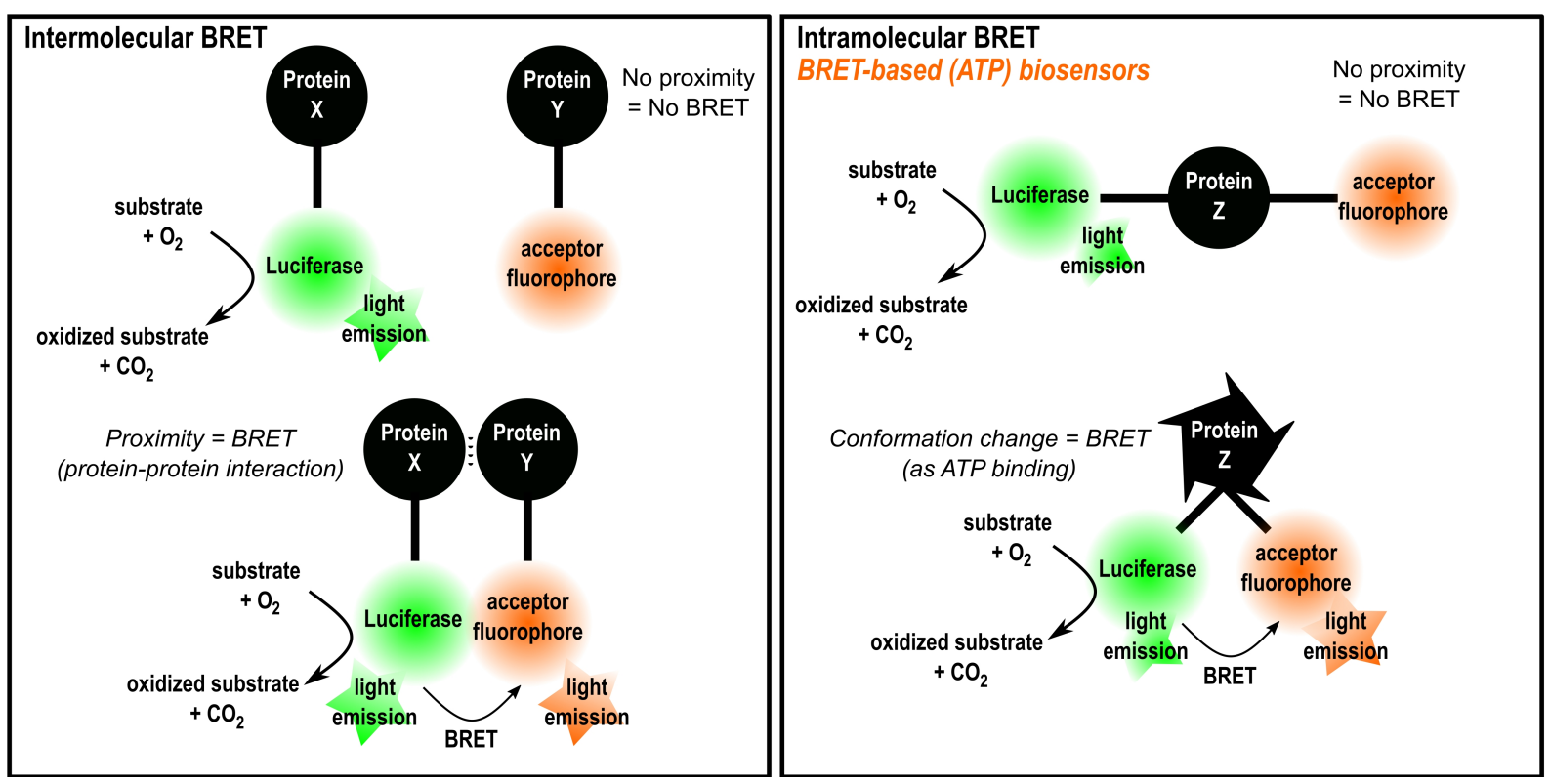

Figure 15. Principle of Bioluminescence Resonance Energy Transfer and BRET-based biosensors

When a luciferase and a fluorophore are close $(\leq 10 \mathrm{~nm})$ the energy derived from a luciferase reaction excites the fluorophore. The fluorophore can, in turn, emit light. Left panel: a putative protein $(X)$ is fused to a luciferase and a second putative protein $(\mathrm{Y})$ is fused to a flurorophore. If $\mathrm{X}$ and $\mathrm{Y}$ proteins do not interact, the BRET phenomenon cannot occur, and the fluorophore cannot emit light. Conversely, the interaction of $X$ and $Y$ allows the BRET phenomenon to occur, and the fluorophore can emit light. Right panel: a putative protein $\mathrm{Z}$ is fused with a luciferase and a flurorophore at each terminus. Conformational folding events allow the BRET phenomenon to occur. This property is used to develop BRET-based biosensors. 
Yoshida et al, developed a BRET version of the ATEAM biosensor (a FRET-based biosensor described in chapter 2.2.5), called BTEAM. This probe consists in the ATP binding domain of the $\varepsilon$ subunit of bacterial ATP synthase flanked by Venus (yellow fluorescent protein, $\lambda \mathrm{em}=528 \mathrm{~nm}$ ) at the $\mathrm{N}$-ter, and a NanoLuciferase which is a non-consuming ATP luciferase at the C-ter. This biosensor was shown to detect intracellular ATP in the cytoplasm (Cyt-BTEAM) or in the mitochondrial matrix (MitBTEAM) in live HeLa cells [82]. Following an analogous approach, Saito et al developed another variant of the ATEAM biosensor, called the Nano-lantern ATP biosensor. This sensor combines BRET and Complementation of Split Luciferase (CSL) [83], and it is built by placing Venus at the N-term of the sensor, while the $\varepsilon$ subunit of the bacterial ATP synthase is inserted within two portions of the NanoLuciferase. When a molecule of ATP binds to the sensor, it allows the reconstitution of a full luciferase, and a BRET reaction between Venus and the Nanoluciferase can occur. This biosensor was validated on plant leaves to study ATP dynamics in the cytoplasm and in chloroplasts. A third BRETbased ATP biosensor designed by Min et al, called ARSeNL ( $\mathrm{m}$ Scarlet-NanoLuc sensor) consists of the $\varepsilon$ subunit of the bacterial ATP synthase inserted between an mScarlet (red fluorescent protein, $\lambda \mathrm{em}=594 \mathrm{~nm}$ ) and a NanoLuciferase [84]. This biosensor was shown to detect ATP levels in a HEK293A cell line and in mice. The major advantage of this biosensor is that it provides a stable and robust signal within animal tissues, and it seems to be particularly useful to study ATP levels in tumours [85].

BRET-based biosensors present many advantages. First, light emission comes from the enzymatic reaction triggered by the addition of the luciferase substrate, which eliminates the need for an external exciting light source (laser). This avoids the generation of autofluorescence and phototoxicity, two phenomena potentially harmful for cells and particularly for pigmented cells such as melanocytes [86]. Moreover, BRET is extremely sensitive because this phenomenon occurs only when luciferase and the fluorophore are close [81]. For measurements on steady-states levels of ATP, this technique can be very useful and easy to perform, especially since luciferin is capable to cross the cellular membrane almost instantaneously and it is not harmful to cell physiology $[77,87]$.

However, BRET also shows an intrinsic limitation due to the luciferin itself. Indeed, some drug excipients such as cyclodextrin, were shown to reduce bioluminescence levels inside cells [88]. In studies focusing on drug development, it is also possible that luciferin might interfere with the substance tested. For all these reasons, the creation of new luciferin variants, luciferases and acceptor fluorophores is a field under intense investigation [89,90]. In addition, the number of cells, the concentration of enzymes and of substrates can severely limit the quantification of BRET. As the light emitted is directly proportional to the amount of ATP in the cell, it is necessary to precisely quantify the initial number of cells. It is also necessary to ensure that the transfection of luciferase for BRETbased biosensors is homogeneous in cells, as the higher the concentration of the biosensor, the higher the amount of the emitted light. Finally, the amount of substrate to integrate into the medium to achieve a maximal detection of ATP must be carefully optimized.

\subsubsection{Single-wavelength ratiometric and intensiometric biosensors}

Another class of biosensors is based on single fluorescent proteins, which exhibit a fluorescence signal or an increase in fluorescence emission at their corresponding wavelengths. This increase occurs only when the molecule of interest, in this case ATP, binds to the sensor, and changes its conformation (Figure 16). The type of fluorophores used is generally circularly-permutated fluorophores. Indeed, circularly-permutated fluorophores have their $\mathrm{N}$ - and C-termini directly fused with a peptide linker, thereby creating new terminal ends close to the chromophore (the fluorophore part responsible for light emission). As a result, the slightest change in conformation is easily detected by the chromophore, which changes its fluorescence properties [91]. 

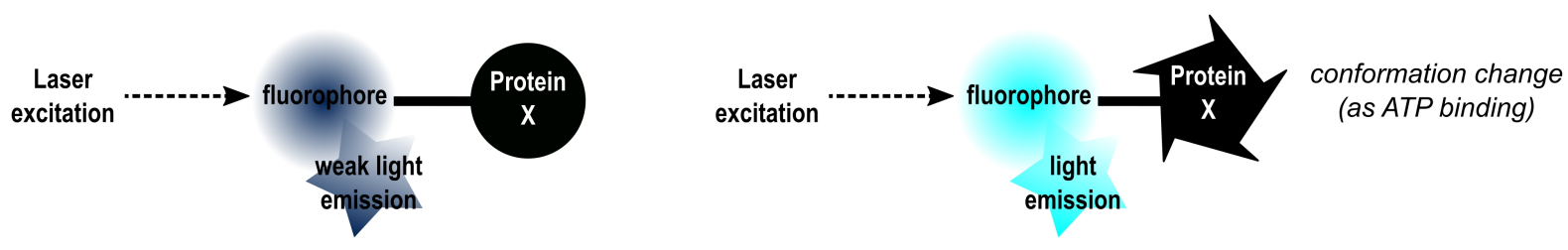

Figure 16. Single wavelength ratiometric and intensiometric biosensors

A putative protein $(X)$ is fused to a circularly-permutated fluorophore. According the conformation of $X$, the fluororophore is capable or not to exhibit a fluorescence emission or an increase in fluorescence emission.

First, the QUEEN biosensor (Quantitative Evaluator of cellular ENergy) is a geneticallyencoded, ratiometric fluorescent ATP indicator that allows to quantify global ATP levels inside bacteria and yeast [92]. This biosensor consists of a single circularly-permutated enhanced GFP $(\lambda$ ex $/$ Aem $=400-494 / 513 \mathrm{~nm})$ inserted between $2 \alpha$-helices of the $\varepsilon$ subunit of bacterial ATP synthase. The integration of circularly-permutated enhanced GFP into a flexible region of a sensory domain, as the $\varepsilon$ subunit, allows to sense slight changes in its conformation. When ATP binds to the biosensor, we observe a spectral shift in the fluorescent properties of the probe, resulting in a shift of the $400 / 494 \mathrm{~nm}$ ratio proportional to the concentration of ATP. Yaginuma et al [92] compared the capacity of the QUEEN biosensor to report on ATP concentration levels with classical luciferase ATP assays in bacterial cells. They showed that the results obtained with the QUEEN biosensor are almost equal to those obtained from bioluminescence luciferase assays.

Second, another ATP biosensor based on fluorescence intensity is iATPSnFR (intensity-based ATP-sensing fluorescent reporter), which allows to monitor extracellular and cytosolic ATP in HEK293 (human embryonic kidney cells) and U373 (Human glioblastoma-astrocytoma cells) [93]. iATPSnFR consists in the insertion of a circularly-permutated superfolder GFP ( $\lambda$ ex/ $/ \mathrm{em}=488 / 525$ $\mathrm{nm}$ ) between $2 \alpha$-helices of the $\varepsilon$ subunit of bacterial ATP synthase as in the QUEEN biosensor. When ATP binds to the biosensor, a rapid increase in fluorescence occurs. To monitor the levels of ATP in specific subcellular compartments, it is also possible to modify this biosensor by adding particular localization signals. However, iATPSnFR shows a modest $\mathrm{pH}$ sensitivity which can be potentially problematic for mitochondria or other subcellular compartments that undergo significant $\mathrm{pH}$ variations.

Third, the intensiometric MaLion family of biosensors (Monitoring aTP Level intensiometric turn-on indicator) was used to follow cytosolic, mitochondrial and nuclear ATP levels in HeLa cells, in primary adipocytes and in Caenorhabditis elegans [94]. The MaLion family of biosensors consists of MaLion $\underline{\mathbb{R}}$ MaLion $\underline{G}$ and MaLion $\underline{B}$ three constructs based on the fusion of the $\varepsilon$ subunit of the bacterial ATP synthase with $\underline{\operatorname{Red}}(\lambda \mathrm{ex} / \lambda \mathrm{em}=565 / 585 \mathrm{~nm})$, Green $(\lambda \mathrm{ex} / \lambda \mathrm{em}=505 / 522 \mathrm{~nm})$ and $\underline{B}$ lue $(\lambda \mathrm{ex} / \lambda \mathrm{em}=373 / 446 \mathrm{~nm})$ fluorophores, respectively. These probes present several advantages: as for iATPSnFR, MaLionB and MaLionG were fused at their N-terminal with nuclear and mitochondrial targeting sequences for organelle-specific ATP estimations. In addition, the higher the concentration of ATP, the greater the fluorescence emission is. Last, all the probes of the family show a very low $\mathrm{pH}$ sensitivity.

Last, the Perceval biosensor family are ratiometric biosensors based on the estimation of the ADP/ATP ratio with live cell imaging. They are composed of a bacterial regulatory protein, GlnK1, linked to circularly permuted Venus $(\lambda e x / \lambda e m=405-490 / 530 \mathrm{~nm})$. Similarly to the $\varepsilon$ subunit of bacterial ATP synthase, GlnK1 undergoes a conformational change when binding ATP. This change induces a change of the $\lambda 490 / \lambda 405 \mathrm{~nm}$ ratio of cpVenus, which is proportional to ATP concentration levels. Conversely, no conformational change is observed upon ADP binding. This biosensor was validated in live mammalian cells and in yeast, and demonstrated its usefulness to determine the energy status and metabolic profiles of cells [95-98]. 


\subsubsection{FRET-based biosensors to measure cellular and extracellular ATP levels}

FRET (Förster's Resonance Energy Transfer) microscopy relies on the transfer of energy between two fluorescent moieties. The two fluorophores are commonly called donor, which is the energy giver, and acceptor, which receives the energy from the donor. As for BRET, the energy transfer occurs only if the donor and acceptor are less than $10 \mathrm{~nm}$ apart and that the emission spectrum of the donor fluorophore overlaps the excitation spectrum of the acceptor fluorophore [99]. This technique is often used to study the physical interaction between two different proteins, and protein oligomerization (intermolecular FRET). In addition, this technique can be also used to study conformational changes of a given protein (intramolecular FRET) and as for BRET-based probes, intramolecular FRET can be used to develop biosensors (Figure 17).
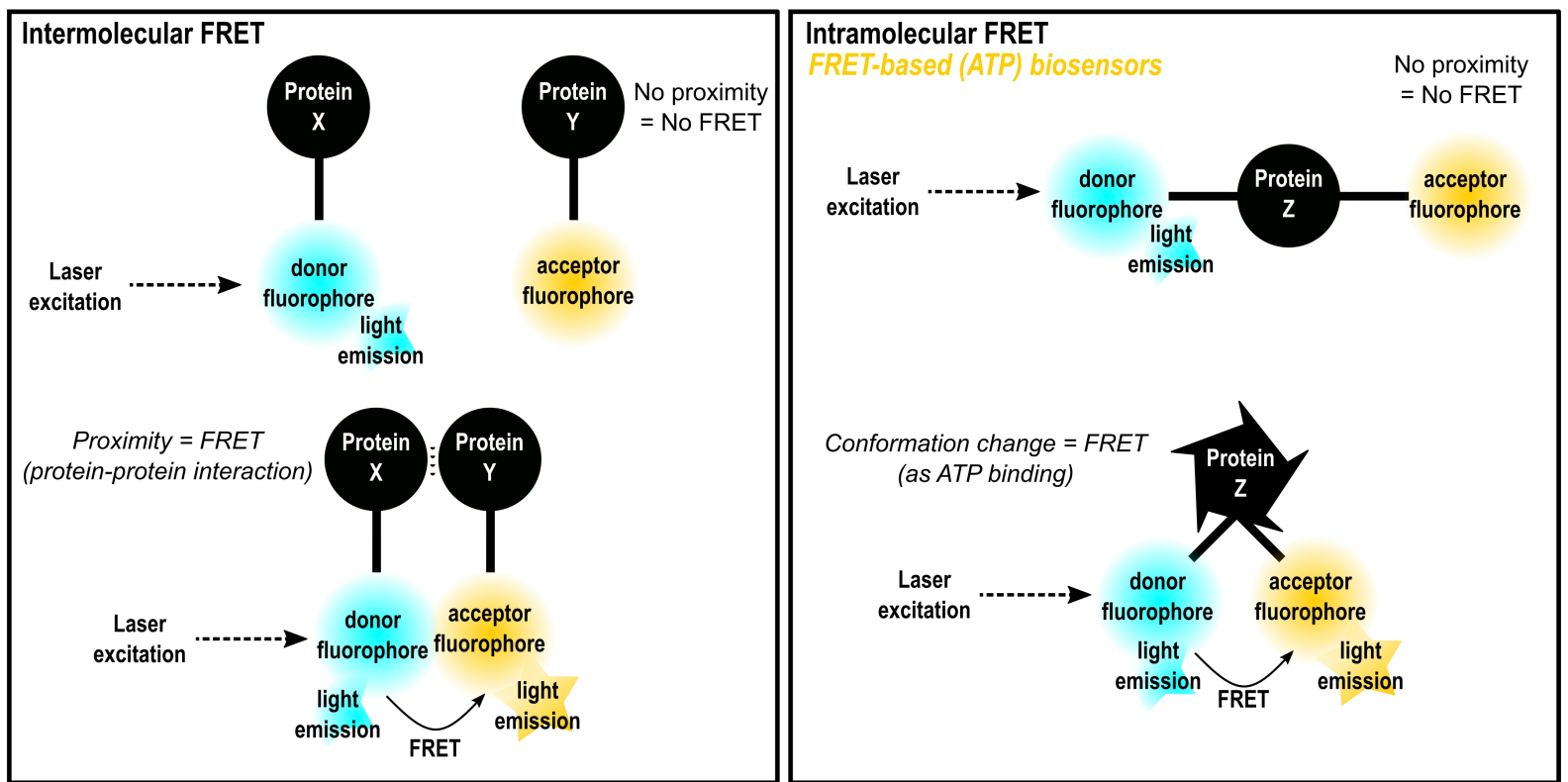

Figure 17. Principle of Fluorescence Resonance Energy Transfer and FRET-based biosensors.

When fluorophores donor and acceptor are closes $(\leq 10 \mathrm{~nm})$ the energy derived from excitation of the donor fluorophore is transmitted to the acceptor fluorophore, which can emits light. Left panel: a protein is fused with a donor fluorophore and a second protein with an acceptor flurorophore. If the proteins do not interact together, the FRET phenomenon cannot occurs and the acceptor fluorophore cannot emit light. Conversly, if the proteins interact together, the FRET phenomenon can occurs and the acceptor fluorophore emit light. Right panel: a unique protein is fused with a donor-acceptor fluorophore pair. The FRET phenomenon car occurs according the protein conformation. This property is used to develop FRET-based biosensors.

A large panel of fluorescent FRET-based ATP biosensors have already been developed. Nowadays, the most diffused probes to monitor ATP in single cells are the ATEAM biosensors (Adenosine 5'-Triphosphate indicator based on Epsilon subunit for Analytical Measurements). Originally developed by Imamura et al, the ATEAM biosensor comprises the $\varepsilon$ subunit of the bacterial ATP synthase within a cyan (mseCFP, $\lambda$ ex/ $/ \mathrm{em}=435 / 475 \mathrm{~nm}$ ) and yellow (cp173-mVenus, $\lambda$ ex/ $/$ em $=515 / 527 \mathrm{~nm}$ ) donor/acceptor pair at the N- and C-termini, respectively [100]. This biosensor allows the monitoring of intracellular ATP in living HeLa cells with a subcellular resolution: ATP was detected in the cytoplasm, nucleus and the mitochondria by adding an organelle-specific localization signal to the biosensor. In the second version of the ATEAM biosensor, called GO-ATEAM, the original donor and acceptor fluorophores were replaced by a green (cp173-mEGFP, $\lambda \mathrm{ex} / \lambda \mathrm{em}=470 / 510$

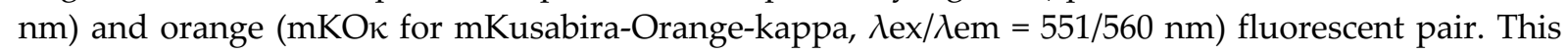
pair allows to have a more stable signal under acidic $\mathrm{pH}$ conditions, and a less phototoxic excitation wavelength in case of long-time observations [101,102]. 
In addition, the optimization of the ATEAM biosensor in an ATEAM1.03NL biosensor, by introducing mutations in the N-term and C-term domains of the $\varepsilon$ subunit, allows the monitoring of intracellular ATP levels at $25^{\circ} \mathrm{C}$. This is compatible with in vivo models as Drosophila melanogaster and Caenorhabditis elegans [103]. By modifying the donor/acceptor pair, the EAF-ATP biosensor (Enhanced Acceptor Fluorescence-based ATP biosensor) was also obtained. It consists of the $\varepsilon$ subunit of bacterial ATP synthase as in the ATEAM biosensors, where the C-term and N-term domains are grafted with green $(\mathrm{GFP}, \lambda \mathrm{ex} / \lambda \mathrm{em}=488 / 507 \mathrm{~nm})$ and yellow $(\mathrm{YFP}, \lambda \mathrm{ex} / \lambda \mathrm{em}=513 / 527 \mathrm{~nm})$ fluorescent proteins, respectively. These fluorophores can be excited at similar wavelengths, which allows the emission of an enhanced fluorescence signal. This biosensor was used to monitor cytoplasmic ATP levels in cells lines derived from glioblastoma in order to better understand the metabolic switch from mitochondrial oxidative phosphorylation to cytoplasmic glycolysis - the Warburg effect - in this model [104].

Last, a re-engineering of the ATEAM biosensor was recently carried out to monitor extracellular ATP. This ecATEAM3.10 biosensor was expressed on the cell surface thanks to a PDGFR transmembrane anchor, with a CFP/YFP donor/acceptor FRET pair (CFP $\lambda$ ex $/ \lambda \mathrm{em}=456 / 480 \mathrm{~nm}$; YFP $\lambda \mathrm{ex} / \lambda \mathrm{em}=513 / 527 \mathrm{~nm}$ ) [105]. As explained previously, ATP is also a key signalling molecule of purinergic signalisation. Therefore, this biosensor is a very useful tool to better understand the dynamics of this nucleotide acting as messenger in the context of synaptic communication.

The FRET technique has several great advantages. First, it allows to work on living cells and at the single-cell level, where studies of molecule dynamics are possible, and has an exquisite spatiotemporal resolution. While canonical fluorescence has a resolution limit around $250 \mathrm{~nm}$, the FRET phenomenon occurs only when fluorophores are less than $10 \mathrm{~nm}$ apart $[99,106]$. In addition, this technique can be performed on standard microscope setups thanks to the great variety of techniques available nowadays to quantify FRET. Among them, ratiometric changes of fluorescence intensity can be easily measured [99]. However, it should also be noted that FRET results could be affected by autofluorescence artifacts and phototoxicity in long-term experiments, which can be resolved by the use of weak phototoxic excitation wavelength as Masahira et al. [101]

Almost unanimously, FRET, BRET and single-wavelength intensiometric/ratiometric biosensors (except for Syn-ATP and the Perceval family biosensors) rely on the conformational changes of a bacterial protein, Bacillus subtilis ATP synthase subunit $\varepsilon$. As described above, the bacterial ATP synthase subunit $\varepsilon$ corresponds to the mitochondrial ATP synthase subunit $\delta$ (Figure 4). This subunit has been thoroughly studied because of its many advantages. First, it is capable of binding ATP without hydrolyzing it [107-109]. Furthermore, the $\varepsilon$ subunit can bind ATP with higher specificity than other nucleotides as ADP, GTP, UTP, and CTP [108]. Then, it undergoes large conformational changes when binding to ATP $[100,110]$. Overall, the use of the $\varepsilon$ subunit of the ATP synthase seems to be a good choice to study intracellular ATP fluctuations. However, it should be noted that this subunit has two main roles within the bacterial ATP synthase: it couples catalysis to proton transport, and it regulates the catalytic activity of the enzyme. The regulations of these essential functions are probably very different from one organism to another, with the difference being more important between bacterial and eukaryotic cells [111]. Therefore, further studies are required to reinforce its relevance to study how ATP is produced in human cells, or its suitability to investigate ATP deregulation in human pathologies [112].

Overall, there is therefore a multitude of tools in the field of luminescence to detect ATP molecule with high selectivity both in cells and in vivo., especially in the field of fluorescent microscopy. Depending on the desired detection method, but also on the model available (i.e. live cells, cell extracts, animal models etc.), it is now possible to find the most suitable sensor for any desired application. On the other hand, rounds of optimization remain to be done in order to extend the use of these tools for diagnostic purposes. 


\section{Preclinical and clinical applications of ATP-monitoring approaches}

\subsection{Mitochondrial pathologies}

Mitochondrial pathologies linked to one or more defects in the activity of the mitochondrial respiratory chain have been broadly documented, and they essentially result from one or more mutations in nuclear DNA or/and in mitochondrial DNA coding for specific subunits of the OXPHOS chain [113-117]. As described above, within the respiratory chain, the subunits of complexes I - III IV and V are encoded both by nuclear and mitochondrial DNA. Only complex II and intramembrane shuttles (coenzyme Q10 and cytochrome C) are entirely encoded by the nuclear genome. Leigh syndrome (subacute necrotizing encephalomyelopathy), LHON (Leber hereditary optic neuropathy), MELAS syndrome (mitochondrial encephalopathy with lactic acidosis and stroke-like episodes) and the NARP syndrome (neuropathy, ataxia and retinitis pigmentosa) are among the best characterized mitochondrial pathologies [116]. In these diseases, it was demonstrated that one or more deficiencies in the mitochondrial respiratory chain subunits irremediably leads to a decrease of ATP production [118].

Currently, the diagnosis of mitochondrial pathologies is carried out by biochemical tests such as analyses of metabolites (lactates, pyruvate, amino acids, etc.) in blood or urine samples, but also respiratory chain enzyme activity assays and oxygen consumption rate estimations. These screens are often correlated with genetic tests, which are carried out on blood lymphocytes or muscle biopsies. To date, around 300 causative genes have been identified in mitochondrial and nuclear DNA to cause mitochondrial diseases [119-121]. In the last steps of diagnosis pipelines, classical clinical tests as electrocardiograms, ophthalmologic examinations, cerebral magnetic resonance imaging are carried out to ascertain the typical clinical manifestations of mitochondrial pathologies (e.g. seizures or slow waves consistent with an encephalopathy can be monitored on electrocardiograms) $[119,122]$.

In a context of mitochondrial disorders diagnostic, Rizza et al developed a biochemical test to measure the mitochondrial ATP production, which recapitulates the overall activity of the mitochondrial respiratory chain [123]. This method requires human skin fibroblasts (from biopsies) and it consists of calculating mitochondrial ATP production through cytosolic NADPH production as a readout (Figure 18).

It is an extremely fast and precise screening method for ATP synthesis in mitochondria, because (i) it determines if there are initial abnormalities in ATP production rates and (ii), by adding intermediate metabolites (succinate, malate, $\alpha$-ketoglutarate, pyruvate) it determines the specific OXPHOS complex affected. Therefore, this tool could be helpful for pre-clinical diagnosis before more invasive interventions, in particular for children, and it helps researchers to directly infer on potential genetic mutations [124-129]. 


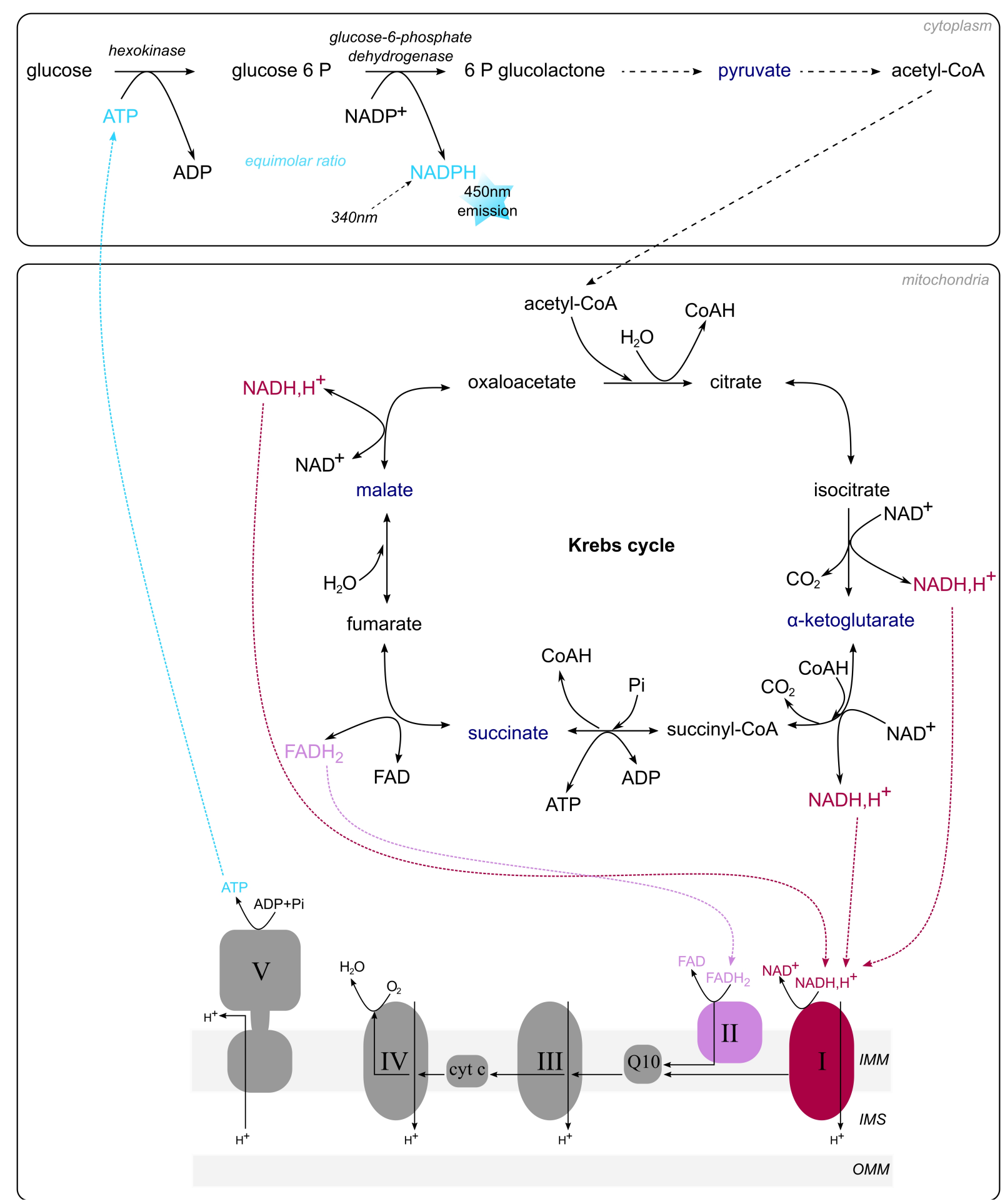

Figure 18. Assaying ATP synthesis in cultured cells via NADPH estimations.

This method is based on the equimolar ratio of NADPH and ATP within the cell (light blue) [123]. NADPH in its reduced form is a fluorescent pyridine nucleotide [130]. This method of measuring ATP levels allows to study the OXPHOS function of mitochondria thanks to the addition of these intermediates within cells (dark blue) and particularly of the complex I (pink) and complex II (purple). 


\subsection{Non-mitochondrial pathologies}

Beyond strict mitochondrial pathologies, it should be noted that an abnormal production of ATP can also be a consequence of pathologies which are not specific to mitochondria. In the section below, we will review some of the most common non-mitochondrial pathologies where defects in ATP production have been detected and where imaging-based methods to screen for ATP levels can be used.

\subsubsection{Chronic glaucoma}

Under the name of chronic glaucoma there is a group of eye diseases caused by damages on the optic nerve. These damages ultimately result in a partial loss of vision and in the long term, they lead to total and irreversible blindness. This can be caused by several factors as genetic predispositions, specific pharmacological treatments such as corticosteroids and the elevation of intraocular pressure [131]. The latter was shown to cause an ATP release from retinal neurons and astrocytes into ocular fluids and retina, as a physiological response to mechanical stimuli $[132,133]$. To date, updated studies should be carried out to explore whether the ATP concentration in the ocular fluids can be considered as a bona fide marker of glaucoma. This analysis could be performed on samples of the aqueous or vitreous humours, obtained after puncture. In one hand, this type of sample would be ideal for diagnostic tools based on luminescence, thanks to the fact that humours are crystalline and do not require spatiotemporal resolution. Although sample collection is invasive to a certain degree, an early diagnosis would definitely limit the destruction of optical fibres in this multifaceted pathology. On the other hand, the use of FRET-based biosensors would be ideal for further studies requiring spatiotemporal resolution, such as studying the mitochondrial network and its ATP production rates in optic nerve cells from glaucoma [134].

\subsubsection{Irritant dermatitis}

Irritant dermatitis is a skin inflammation caused by contact with irritating chemicals as lactic acid, isopropanol or sodium hydroxyde. This innate inflammatory response manifests as itching, pain, redness, swelling, and papules on the skin. At the molecular level, the release of ATP into the extracellular medium is among the most prominent proinflammatory features [135-137]. Therefore, monitoring the production and/or the release of ATP by skin cells is an excellent way to determine if a chemical is dangerous or not. This would be very useful in the medical field (for antiseptics, pomades etc...), in the cosmetics field (for perfumes, creams etc...) or even in the agricultural field (for pesticides, animal medicines...). In their study, Mizumoto et al. used the supernatant obtained from human primary keratinocytes previously treated with different chemicals. To measure ATP concentrations released from keratinocytes in the medium, they used a commercialized luciferinluciferase assay, a method readily usable in the vast majority of laboratories, also employable for highthroughput screening purposes [137].

\subsubsection{Human Immunodeficiency Virus (HIV) - Associated Neurocognitive Disorders}

HIV-Associated Neurocognitive Disorders (HAND) are cognitive impairments present in $20 \%-50 \%$ of people infected with HIV, and commonly known as People Living With HIV (PLWH) [138]. HAND divide into 3 categories: HIV-1-associated asymptomatic neurocognitive impairment (ANI), HIV-1-associated mild neurocognitive disorder (MND) and HIV-1-associated dementia (HAD) [139]. Antiretroviral therapy (ART) represents a dramatic advance for the lifespan and the quality of life of PLWH. However, the symptoms of HAND continue to affect the daily lives of patients, as a persistent neuroinflammation in the brain causes synaptic dysfunctions and neuronal death [140]. 
Very recent studies have demonstrated the ability of circulating ATP to be a predictive biomarker of HAND [141,142]. In detail, Pannexin-1 (PANX-1) is an ATP-permeable channel linked to viral cell entry. Its opening, in the context of a viral infection, causes the release of ATP into the extracellular space. People with HAND were found to have spontaneous openings of these channels and a higher level of circulating ATP. In order to measure circulating ATP, Velasquez et al. used PLWH serum and plasma samples, and performed bioluminescence-based ATP quantifications. These approaches not only bring new perspectives for a possible early diagnosis, but also future therapeutics targeting the opening rate of PANX-1 channels [141].

\subsubsection{Pathologies related to blood vessels}

As ATP is released into the bloodstream as a vascular response to stress or injury, its signalling function is particularly relevant in pathologies related to blood vessels [143]. Red blood cells (RBCs) modulate vasodilation by releasing ATP when oxygen concentrations decrease $[144,145]$. Therefore, the level of extracellular ATP increases in the case of infections. It is the case of sepsis, a generalized inflammatory response caused by a severe bacterial infection. A recent study in a model of sepsis showed that an increase of extracellular ATP parallels a significant decrease in levels of intracellular ATP [146]. Li et al quantified the intracellular ATP levels in RBCs by adding lipopolysaccharides, bacterial endotoxins, or under condition of therapeutic treatment with tigecycline, an antibiotic used against sepsis. Then, they evaluated intracellular ATP levels using HPLC and they provided evidence that it can be used as a biomarker for rapid diagnosis of sepsis. However, further studies are required as sepsis is a multi-factor disease and to gain a spatiotemporal view of the infection process [146].

More surprisingly, Suardi et al. demonstrated the therapeutic properties of laser wavelengths. Indeed, RBC exposure to a precise wavelength $(460 \mathrm{~nm}$ or $532 \mathrm{~nm})$ for a few seconds (50s and $40 \mathrm{~s}$, respectively) increases the viability of RBCs by stimulating ATPase signalling pathways $[147,148]$, in particular $\mathrm{Na}^{+}, \mathrm{K}^{+}$-ATPase [149] and by increasing intracellular ATP levels. This study was performed on RBCs from patients with anemia, and ATP levels were measured using commercialized luminescence-based kits [150]. It would be fascinating to speculate that it is enough to irradiate blood samples from anemic patients and inject them back to see a positive effect on the outcome of the disease. However, it is hardly usable to implement therapeutic options in patients, as irradiation of cells systematically causes a wide series of damages on living cells [151].

Last, cardiac ischemia is caused by insufficient blood flow in the heart. As a result, the heart has insufficient oxygen supply and toxic molecules (resulting from anaerobic metabolism) are no longer eliminated, which can lead to necrosis of the heart tissue. A relevant study has shown that the metabolites derived from ATP catabolism - inosine and hypoxanthine - and found in blood plasma can also be used as biomarkers of cardiac ischemia using HPLC [152]. This paves the way for early diagnosis using non-invasive methods that could prevent the necrosis of the heart tissue.

\subsubsection{Cancer}

Many studies have investigated the role of ATP in the development, progression and maintenance of cancer cells [153]. Although the detailed description of cancer-related metabolic alterations are not in the scope of the present review and have been discussed elsewhere [154,155], it deserves to be mentioned that the tumour microenvironment contains a high concentration of ATP, which is then released into the extracellular environment in an uncontrolled manner by cancer cells. In addition, cancer cells have the ability to absorb extracellular ATP and use it to activate molecular programs for tumour progression $[156,157]$. The study of the tumoral release of ATP, its role and its mechanisms of action are a hot topic to ameliorate current anticancer therapies [158]. Among cancer- 
related metabolic alterations [154,155], the Warburg effect is one of the most studied ones [159]. Recent evidence proposes that this process is an epiphenomenon parallel to tumorigenesis [160]. This is why the Warburg effect continues to be studied, in particular through the use of FRET-based biosensors which can be used in vivo [104]. Thanks to their high sensitivity and their capacity to establish spaciotemporal resolution inside tumours, the use of fluorescence techniques is essential to fully understand how the Warburg effect is in corsstalk with tumorigenesis, but also to develop new therapeutic approaches.

\subsection{Additional applications}

Beyond the interest of ATP as a biomarker in disease paradigms, monitoring its levels is also a valuable tool to determine the degree of contamination of surfaces in healthcare-related environments [161-165]. Preventing healthcare-associated infections is mandatory to limit the contamination of people in hospitals and who are weakened by various pathologies. A large majority of the approaches to monitor the contamination of surfaces are based on the luciferin-luciferase bioluminescence technique, thanks to the ability of bacteria present on surfaces to perform the luminescence reaction [166]. Therefore, ATP levels can be directly correlated with the number of Colony-Forming Units (CFU) per mL. This type of monitoring makes it possible to improve current surface decontamination protocols, and to implement new ones in the near future [167].

\section{Conclusion and perspectives}

As presented here, there is a wide range of tools available to detect and / or quantify ATP levels. Historically, the first techniques implemented were biophysical approaches, such as NMR, HPLC and LC-MS/MS. These techniques have a very precise readout and allow the detection of ATP levels in denatured samples (blood, cell extracts, mitochondrial extracts) that require a high quantity of starting material. However, these are extremely expensive and this type of analysis can hardly be carried out in conventional diagnostic and/or research laboratories. There are also biochemical techniques such as respirometry, which can indirectly measure the amount of ATP produced within mitochondria by linking ATP production to oxygen consumption in live intact cells or mitochondrial isolates. Although the possibility of using live cells is a great advance to study the concentration of ATP in its physiological environment, respirometry does not provide insights on its spatiotemporal variations. In this light, the arrival of luminescence and fluorescence-based sensors has revolutionized the research on ATP. The biggest benefit of these tools is that we can finally achieve the spatiotemporal resolution of ATP levels at the single-cell scale. Since ATP is largely synthesized within mitochondria, the use of an ATP synthase subunit as a mean of sensing ATP is particularly convenient. However, it should be remembered that the biosensors available rely on a bacterial subunit of the ATP synthase, and not a mammalian one. This could raise questions of the physiological relevance of these probes to study ATP fluctuations in eukaryotic cells and in animal models.

Despite the risks of photobleaching, FRET-based biosensors appear to be the most promising class of probes, as they possess a superior spatiotemporal resolution thanks to the FRET phenomenon and provide reliable quantitative information on subcellular ATP levels. However, they need to be stably or transiently integrated in the genome of the targeted cell. Chemosensors and electrochemosensors do not require an integration, but are not easy to use since the sole parameter of mitochondrial membrane potential turned out to be insufficient for mitochondrial targeting. Single wavelength-bases biosensors are biosensors with on/off readouts, but they were shown to present a fluorescent background which makes it non-trivial to determine the off state. However, they are suitable to detect large variations in ATP production. Finally, BRET-based biosensors have a spatiotemporal resolution similar to FRET probes, and do not require an external light source (i.e., a laser) to trigger the luciferin-luciferase reaction. In contrast to the large body of literature 
demonstrating the phototoxic effects of laser excitations and wavelengths on cell viability, and the response of organelles to irradiations, there are very few studies exploring the effects of luciferin on cell physiology. Unless the toxic effects of luciferin are formally ruled out in future studies, FRETbased probes currently represent the best and most extensively characterized sets of probes to monitor ATP in living cells and with spatiotemporal resolution.

\section{Author Contributions}

S.L.N participated in the conceptualization of this review and wrote the original draft; G.B. co-conceptualized the manuscript, supervised and reviewed the manuscript.

\section{Funding}

This work was supported by the Centre National de la Recherche Scientifique (CNRS). S.L.N. was supported by a $\mathrm{PhD}$ fellowship from the Association Nationale Recherche Technologie (ANRT) and LVMH. Funders have no role in manuscript preparation or revision.

\section{Acknowledgments}

We thank Elif Begüm Gökerküçük for helpful discussions and support.

\section{Conflicts of Interest}

The authors declare no conflict of interest.

\section{References}

[1] Lipmann F. The roots of bioenergetics. Ciba Found Symp 1975:3-22. https://doi.org/10.1002/9780470720134.ch2.

[2] Dunn J, Grider MH. Physiology, Adenosine Triphosphate. StatPearls, Treasure Island (FL): StatPearls Publishing; 2020.

[3] Wangler A, Schmidt C, Sadowski G, Held C. Standard Gibbs Energy of Metabolic Reactions: III The 3-Phosphoglycerate Kinase Reaction. ACS Omega 2018;3:1783-90. https://doi.org/10.1021/acsomega.7b01704.

[4] Pivovarov AS, Calahorro F, Walker RJ. Na+/K+-pump and neurotransmitter membrane receptors. Invert Neurosci 2018;19:1. https://doi.org/10.1007/s10158-018-0221-7.

[5] Klenchin VA, Martin TF. Priming in exocytosis: attaining fusion-competence after vesicle docking. Biochimie 2000;82:399-407. https://doi.org/10.1016/s0300-9084(00)00208-x.

[6] Pathak D, Shields LY, Mendelsohn BA, Haddad D, Lin W, Gerencser AA, et al. The Role of Mitochondrially Derived ATP in Synaptic Vesicle Recycling. J Biol Chem 2015;290:22325-36. https://doi.org/10.1074/jbc.M115.656405.

[7] Sugi H. Molecular Mechanism of ATP-Dependent Actin-Myosin Interaction in Muscle Contraction. Jpn J Physiol 1993;43:435-54. https://doi.org/10.2170/jjphysiol.43.435.

[8] Bonora M, Patergnani S, Rimessi A, De Marchi E, Suski JM, Bononi A, et al. ATP synthesis and storage. Purinergic Signal 2012;8:343-57. https://doi.org/10.1007/s11302-012-9305-8.

[9] Schwiebert EM, Zsembery A. Extracellular ATP as a signaling molecule for epithelial cells. Biochim Biophys Acta 2003;1615:7-32. https://doi.org/10.1016/s0005-2736(03)00210-4.

[10] Idzko M, Ferrari D, Eltzschig HK. Nucleotide signalling during inflammation. Nature 2014;509:310-7. https://doi.org/10.1038/nature13085.

[11] Akter S, Sharma RK, Sharma S, Rastogi S, Fiebich BL, Akundi RS. Exogenous ATP modulates PGE2 release in macrophages through sustained phosphorylation of CDK9 and p38 MAPK. J Leukoc Biol n.d.;n/a. https://doi.org/10.1002/JLB.3A1219-697RR.

[12] Novak I. ATP as a signaling molecule: the exocrine focus. News Physiol Sci Int J Physiol Prod Jointly Int Union Physiol Sci Am Physiol Soc 2003;18:12-7. https://doi.org/10.1152/nips.01409.2002.

[13] Burnstock G. Purinergic signalling: past, present and future. Braz J Med Biol Res 2009;42:3-8. https://doi.org/10.1590/S0100-879X2008005000037. 
[14] Gout E, Rébeillé F, Douce R, Bligny R. Interplay of Mg2+, ADP, and ATP in the cytosol and mitochondria: Unravelling the role of $\mathrm{Mg} 2+$ in cell respiration. Proc Natl Acad Sci 2014;111:E4560-7. https://doi.org/10.1073/pnas.1406251111.

[15] du Plessis SS, Agarwal A, Mohanty G, van der Linde M. Oxidative phosphorylation versus glycolysis: what fuel do spermatozoa use? Asian J Androl 2015;17:230-5. https://doi.org/10.4103/1008-682X.135123.

[16] Signes A, Fernandez-Vizarra E. Assembly of mammalian oxidative phosphorylation complexes I-V and supercomplexes. Essays Biochem 2018;62:255-70. https://doi.org/10.1042/EBC20170098.

[17] Carroll J, Fearnley IM, Skehel JM, Shannon RJ, Hirst J, Walker JE. Bovine complex I is a complex of 45 different subunits. J Biol Chem 2006;281:32724-7. https://doi.org/10.1074/jbc.M607135200.

[18] Whitehouse DG. Respiratory Chain and ATP Synthase n.d.:6.

[19] Crane FL. Biochemical Functions of Coenzyme Q10. J Am Coll Nutr 2001;20:591-8. https://doi.org/10.1080/07315724.2001.10719063.

[20] Scott Mathews F. The structure, function and evolution of cytochromes. Prog Biophys Mol Biol 1985;45:1-56. https://doi.org/10.1016/0079-6107(85)90004-5.

[21] Martijn J, Vosseberg J, Guy L, Offre P, Ettema TJG. Deep mitochondrial origin outside the sampled alphaproteobacteria. Nature 2018;557:101-5. https://doi.org/10.1038/s41586-018-0059-5.

[22] Gabaldón T. Relative timing of mitochondrial endosymbiosis and the "pre-mitochondrial symbioses" hypothesis. IUBMB Life 2018;70:1188-96. https://doi.org/10.1002/iub.1950.

[23] Gray MW. Lynn Margulis and the endosymbiont hypothesis: 50 years later. Mol Biol Cell 2017;28:1285-7. https://doi.org/10.1091/mbc.E16-07-0509.

[24] Roger AJ, Muñoz-Gómez SA, Kamikawa R. The Origin and Diversification of Mitochondria. Curr Biol CB 2017;27:R1177-92. https://doi.org/10.1016/j.cub.2017.09.015.

[25] Gray MW. Mitochondrial Evolution. Cold Spring Harb Perspect Biol $2012 ; 4$. https://doi.org/10.1101/cshperspect.a011403.

[26] Gray MW. The pre-endosymbiont hypothesis: a new perspective on the origin and evolution of mitochondria. Cold Spring Harb Perspect Biol 2014;6. https://doi.org/10.1101/cshperspect.a016097.

[27] Morelli A, Rosano C. A True Symbiosis for the Mitochondria Evolution. Bioenerg Open Access 2016;05. https://doi.org/10.4172/2167-7662.1000137.

[28] Guo H, Suzuki T, Rubinstein JL. Structure of a bacterial ATP synthase. ELife 2019;8. https://doi.org/10.7554/eLife.43128.

[29] Dabbeni-Sala F, Rai AK, Lippe G. F0F1 ATP Synthase: A Fascinating Challenge for Proteomics. Proteomics - Hum Dis Protein Funct 2012. https://doi.org/10.5772/31082.

[30] He J, Ford HC, Carroll J, Douglas C, Gonzales E, Ding S, et al. Assembly of the membrane domain of ATP synthase in human mitochondria. Proc Natl Acad Sci 2018;115:2988-93. https://doi.org/10.1073/pnas.1722086115.

[31] Xu T, Pagadala V, Mueller DM. Understanding structure, function, and mutations in the mitochondrial ATP synthase. Microb Cell Graz Austria 2015;2:105-25. https://doi.org/10.15698/mic2015.04.197.

[32] Menz RI, Walker JE, Leslie AG. Structure of bovine mitochondrial F(1)-ATPase with nucleotide bound to all three catalytic sites: implications for the mechanism of rotary catalysis. Cell 2001;106:331-41. https://doi.org/10.1016/s0092-8674(01)00452-4.

[33] Habersetzer J, Ziani W, Larrieu I, Stines-Chaumeil C, Giraud M-F, Brèthes D, et al. ATP synthase oligomerization: from the enzyme models to the mitochondrial morphology. Int J Biochem Cell Biol 2013;45:99-105. https://doi.org/10.1016/j.biocel.2012.05.017.

[34] Wittig I, Meyer B, Heide H, Steger M, Bleier L, Wumaier Z, et al. Assembly and oligomerization of human ATP synthase lacking mitochondrial subunits a and A6L. Biochim Biophys Acta 2010;1797:1004-11. https://doi.org/10.1016/j.bbabio.2010.02.021. 
[35] Mlynárik V. Introduction to nuclear magnetic resonance. Anal Biochem 2017;529:4-9. https://doi.org/10.1016/j.ab.2016.05.006.

[36] Sersa I, Kranjc S, Sersa G, Nemec-Svete A, Lozar B, Sepe A, et al. Study of radiation induced changes of phosphorus metabolism in mice by (31)P NMR spectroscopy. Radiol Oncol 2010;44:174-9. https://doi.org/10.2478/v10019-010-0030-z.

[37] Guo B, Gurel PS, Shu R, Higgs HN, Pellegrini M, Mierke DF. Monitoring ATP hydrolysis and ATPase inhibitor screening using 1H NMR. Chem Commun Camb Engl 2014;50:12037-9. https://doi.org/10.1039/c4cc04399e.

[38] Lian Y, Jiang H, Feng J, Wang X, Hou X, Deng P. Direct and simultaneous quantification of ATP, ADP and AMP by (1)H and (31)P Nuclear Magnetic Resonance spectroscopy. Talanta 2016;150:485-92. https://doi.org/10.1016/j.talanta.2015.12.051.

[39] Kudisch B, Maiuri M, Moretti L, Oviedo MB, Wang L, Oblinsky DG, et al. Ring currents modulate optoelectronic properties of aromatic chromophores at 25 T. Proc Natl Acad Sci 2020;117:11289-98. https://doi.org/10.1073/pnas.1918148117.

[40] Parker T, Limer E, Watson AD, Defernez M, Williamson D, Kemsley EK. 60 MHz 1H NMR spectroscopy for the analysis of edible oils. Trends Anal Chem 2014;57:147-58. https://doi.org/10.1016/j.trac.2014.02.006.

[41] Quine RW, Rinard GA, Shi Y, Buchanan L, Biller JR, Eaton SS, et al. UHF EPR spectrometer operating at frequencies between $400 \mathrm{MHz}$ and $1 \mathrm{GHz}$. Concepts Magn Reson Part B Magn Reson Eng 2016;46B:123-33. https://doi.org/10.1002/cmr.b.21328.

[42] Holzgrabe U, Deubner R, Schollmayer C, Waibel B. Quantitative NMR spectroscopyApplications in drug analysis. J Pharm Biomed Anal 2005;38:806-12. https://doi.org/10.1016/j.jpba.2005.01.050.

[43] Trams EG, Kaufmann H, Burnstock G. A proposal for the role of ecto-enzymes and adenylates in traumatic shock. J Theor Biol 1980;87:609-21. https://doi.org/10.1016/0022-5193(80)90239-8.

[44] Gorman MW, Feigl EO, Buffington CW. Human plasma ATP concentration. Clin Chem 2007;53:318-25. https://doi.org/10.1373/clinchem.2006.076364.

[45] Blum F. High performance liquid chromatography. Br J Hosp Med Lond Engl 2005 2014;75:C18-21. https://doi.org/10.12968/hmed.2014.75.Sup2.C18.

[46] Liu H, Jiang Y, Luo Y, Jiang W. A Simple and Rapid Determination of ATP, ADP and AMP Concentrations in Pericarp Tissue of Litchi Fruit by High Performance Liquid Chromatography 2006:4.

[47] Menegollo M, Tessari I, Bubacco L, Szabadkai G. Determination of ATP, ADP, and AMP Levels by Reversed-Phase High-Performance Liquid Chromatography in Cultured Cells. Methods Mol Biol Clifton NJ 2019;1925:223-32. https://doi.org/10.1007/978-1-4939-9018-4_19.

[48] von Papen M, Gambaryan S, Schütz C, Geiger J. Determination of ATP and ADP Secretion from Human and Mouse Platelets by an HPLC Assay. Transfus Med Hemotherapy Off Organ Dtsch Ges Transfusionsmedizin Immunhamatologie 2013;40:109-16. https://doi.org/10.1159/000350294.

[49] Bhatt DP, Chen X, Geiger JD, Rosenberger TA. A sensitive HPLC-based method to quantify adenine nucleotides in primary astrocyte cell cultures. J Chromatogr B Analyt Technol Biomed Life Sci 2012;889-890:110-5. https://doi.org/10.1016/j.jchromb.2012.02.005.

[50] Pitt JJ. Principles and Applications of Liquid Chromatography-Mass Spectrometry in Clinical Biochemistry. Clin Biochem Rev 2009;30:19-34.

[51] Fu X, Deja S, Kucejova B, Duarte JAG, McDonald JG, Burgess SC. Targeted Determination of Tissue Energy Status by LC-MS/MS. Anal Chem 2019;91:5881-7. https://doi.org/10.1021/acs.analchem.9b00217.

[52] Kim H, Kosinski P, Kung C, Dang L, Chen Y, Yang H, et al. A fit-for-purpose LC-MS/MS method for the simultaneous quantitation of ATP and 2,3-DPG in human K2EDTA whole blood. J Chromatogr B Analyt Technol Biomed Life Sci 2017;1061-1062:89-96. https://doi.org/10.1016/j.jchromb.2017.07.010. 
[53] Djafarzadeh S, Jakob SM. High-resolution Respirometry to Assess Mitochondrial Function in Permeabilized and Intact Cells. J Vis Exp JoVE 2017. https://doi.org/10.3791/54985.

[54] Yamamoto H, Morino K, Mengistu L, Ishibashi T, Kiriyama K, Ikami T, et al. Amla Enhances Mitochondrial Spare Respiratory Capacity by Increasing Mitochondrial Biogenesis and Antioxidant Systems in a Murine Skeletal Muscle Cell Line. Oxid Med Cell Longev 2016;2016. https://doi.org/10.1155/2016/1735841.

[55] Devenish RJ, Prescott M, Boyle GM, Nagley P. The oligomycin axis of mitochondrial ATP synthase: OSCP and the proton channel. J Bioenerg Biomembr 2000;32:507-15. https://doi.org/10.1023/a:1005621125812.

[56] Benz R, McLaughlin S. The molecular mechanism of action of the proton ionophore FCCP (carbonylcyanide p-trifluoromethoxyphenylhydrazone). Biophys J 1983;41:381-98. https://doi.org/10.1016/S0006-3495(83)84449-X.

[57] Ma X, Jin M, Cai Y, Xia H, Long K, Liu J, et al. Mitochondrial Electron Transport Chain Complex III Is Required for Antimycin A to Inhibit Autophagy. Chem Biol 2011;18:1474-81. https://doi.org/10.1016/j.chembiol.2011.08.009.

[58] Heinz S, Freyberger A, Lawrenz B, Schladt L, Schmuck G, Ellinger-Ziegelbauer H. Mechanistic Investigations of the Mitochondrial Complex I Inhibitor Rotenone in the Context of Pharmacological and Safety Evaluation. Sci Rep 2017;7. https://doi.org/10.1038/srep45465.

[59] Blazek M, Santisteban TS, Zengerle R, Meier M. Analysis of fast protein phosphorylation kinetics in single cells on a microfluidic chip. Lab Chip 2015;15:726-34. https://doi.org/10.1039/c4lc00797b.

[60] Kobayashi H, Ogawa M, Alford R, Choyke PL, Urano Y. New strategies for fluorescent probe design in medical diagnostic imaging. Chem Rev 2010;110:2620-40. https://doi.org/10.1021/cr900263j.

[61] Wu Y, Wen J, Li H, Sun S, Xu Y. Fluorescent probes for recognition of ATP. Chin Chem Lett 2017;28:1916-24. https://doi.org/10.1016/j.cclet.2017.09.032.

[62] Liu Y, Lei J, Huang Y, Ju H. "Off-on" electrochemiluminescence system for sensitive detection of ATP via target-induced structure switching. Anal Chem 2014;86:8735-41. https://doi.org/10.1021/ac501913c.

[63] Liu B, Cui Y, Tang D, Yang H, Chen G. Au(III)-assisted core-shell iron oxide@poly(ophenylenediamine) nanostructures for ultrasensitive electrochemical aptasensors based on DNase I-catalyzed target recycling. Chem Commun 2012;48:2624-6. https://doi.org/10.1039/C2CC17790K.

[64] Yin B-C, Guan Y-M, Ye B-C. An ultrasensitive electrochemical DNA sensor based on the ssDNA-assisted cascade of hybridization reaction. Chem Commun 2012;48:4208-10. https://doi.org/10.1039/C2CC30997A.

[65] Ho Y-P, Leong KW. Quantum dot-based theranostics. Nanoscale 2010;2:60-8. https://doi.org/10.1039/b9nr00178f.

[66] Pleskova S, Mikheeva E, Gornostaeva E. Using of Quantum Dots in Biology and Medicine. Adv Exp Med Biol 2018;1048:323-34. https://doi.org/10.1007/978-3-319-72041-8_19.

[67] Jaiswal JK, Goldman ER, Mattoussi H, Simon SM. Use of quantum dots for live cell imaging. Nat Methods 2004;1:73-8. https://doi.org/10.1038/nmeth1004-73.

[68] Xu Z, Zeng G, Liu Y, Zhang X, Cheng J, Zhang J, et al. Monitoring mitochondrial ATP in live cells: An ATP multisite-binding fluorescence turn-on probe. Dyes Pigments 2019;163:559-63. https://doi.org/10.1016/j.dyepig.2018.12.035.

[69] Reungpatthanaphong P, Dechsupa S, Meesungnoen J, Loetchutinat C, Mankhetkorn S. Rhodamine $\mathrm{B}$ as a mitochondrial probe for measurement and monitoring of mitochondrial membrane potential in drug-sensitive and -resistant cells. J Biochem Biophys Methods 2003;57:1-16. https://doi.org/10.1016/s0165-022x(03)00032-0. 
[70] Wang L, Yuan L, Zeng X, Peng J, Ni Y, Er JC, et al. A Multisite-Binding Switchable Fluorescent Probe for Monitoring Mitochondrial ATP Level Fluctuation in Live Cells. Angew Chem Int Ed Engl 2016;55:1773-6. https://doi.org/10.1002/anie.201510003.

[71] Tan K-Y, Li C-Y, Li Y-F, Fei J, Yang B, Fu Y-J, et al. Real-Time Monitoring ATP in Mitochondrion of Living Cells: A Specific Fluorescent Probe for ATP by Dual Recognition Sites. Anal Chem 2017;89:1749-56. https://doi.org/10.1021/acs.analchem.6b04020.

[72] Liu Y, Lee D, Wu D, Swamy KMK, Yoon J. A new kind of rhodamine-based fluorescence turnon probe for monitoring ATP in mitochondria. Sens Actuators B Chem 2018;265:429-34. https://doi.org/10.1016/j.snb.2018.03.081.

[73] Gao J-Y, Huang W-C, Huang P-Y, Song C-Y, Hong J-L. Light-Up of Rhodamine Hydrazide to Generate Emissive Initiator for Polymerization and to Afford Photochromic Polypeptide Metal Complex. Polymers 2017;9. https://doi.org/10.3390/polym9090419.

[74] Inouye S. Firefly luciferase: an adenylate-forming enzyme for multicatalytic functions. Cell Mol Life Sci CMLS 2010;67:387-404. https://doi.org/10.1007/s00018-009-0170-8.

[75] Holmsen H, Storm E, Day HJ. Determination of ATP and ADP in blood platelets: a modification of the firefly luciferase assay for plasma. Anal Biochem 1972;46:489-501. https://doi.org/10.1016/0003-2697(72)90323-5.

[76] Kimmich GA, Randles J, Brand JS. Assay of picomole amounts of ATP, ADP, and AMP using the luciferase enzyme system. Anal Biochem 1975;69:187-206. https:/doi.org/10.1016/00032697(75)90580-1.

[77] Morciano G, Sarti AC, Marchi S, Missiroli S, Falzoni S, Raffaghello L, et al. Use of luciferase probes to measure ATP in living cells and animals. Nat Protoc 2017;12:1542-62. https://doi.org/10.1038/nprot.2017.052.

[78] Rangaraju V, Calloway N, Ryan TA. Activity-driven local ATP synthesis is required for synaptic function. Cell 2014;156:825-35. https://doi.org/10.1016/j.cell.2013.12.042.

[79] Min K, Steghens J. ADP is produced by firefly luciferase but its synthesis is independent of the light emitting properties. Biochimie 2001;83:523-8. https://doi.org/10.1016/s0300-9084(01)012767.

[80] Lemasters JJ, Hackenbrock CR. [4] Firefly luciferase assay for ATP production by mitochondria. Methods Enzymol., vol. 57, Academic Press; 1978, p. 36-50. https://doi.org/10.1016/00766879(78)57006-7.

[81] Brown NE, Blumer JB, Hepler JR. Bioluminescence Resonance Energy Transfer to Detect Protein-Protein Interactions in Live Cells. Methods Mol Biol Clifton NJ 2015;1278:457-65. https://doi.org/10.1007/978-1-4939-2425-7_30.

[82] Yoshida T, Kakizuka A, Imamura H. BTeam, a Novel BRET-based Biosensor for the Accurate Quantification of ATP Concentration within Living Cells. Sci Rep 2016;6:39618. https://doi.org/10.1038/srep39618.

[83] Saito K, Chang Y-F, Horikawa K, Hatsugai N, Higuchi Y, Hashida M, et al. Luminescent proteins for high-speed single-cell and whole-body imaging. Nat Commun 2012;3:1262. https://doi.org/10.1038/ncomms2248.

[84] Min S-H, French AR, Trull KJ, Tat K, Varney SA, Tantama M. Ratiometric BRET Measurements of ATP with a Genetically-Encoded Luminescent Sensor. Sensors 2019;19. https://doi.org/10.3390/s19163502.

[85] Dagogo-Jack I, Shaw AT. Tumour heterogeneity and resistance to cancer therapies. Nat Rev Clin Oncol 2018;15:81-94. https://doi.org/10.1038/nrclinonc.2017.166.

[86] Arts R, Aper SJA, Merkx M. Engineering BRET-Sensor Proteins. Methods Enzymol 2017;589:87-114. https://doi.org/10.1016/bs.mie.2017.01.010.

[87] Ignowski JM, Schaffer DV. Kinetic analysis and modeling of firefly luciferase as a quantitative reporter gene in live mammalian cells. Biotechnol Bioeng 2004;86:827-34. https://doi.org/10.1002/bit.20059. 
[88] Kumar JS, Miller Jenkins LM, Gottesman MM, Hall MD. The Drug Excipient Cyclodextrin Interacts With d-Luciferin and Interferes With Bioluminescence Imaging. Mol Imaging 2016;15. https://doi.org/10.1177/1536012115625225.

[89] Takakura H, Sasakura K, Ueno T, Urano Y, Terai T, Hanaoka K, et al. Development of luciferin analogues bearing an amino group and their application as BRET donors. Chem Asian J 2010;5:2053-61. https://doi.org/10.1002/asia.201000219.

[90] Weihs F, Dacres H. Red-shifted bioluminescence Resonance Energy Transfer: Improved tools and materials for analytical in vivo approaches. TrAC Trends Anal Chem 2019;116:61-73. https://doi.org/10.1016/j.trac.2019.04.011.

[91] Kostyuk AI, Demidovich AD, Kotova DA, Belousov VV, Bilan DS. Circularly Permuted Fluorescent Protein-Based Indicators: History, Principles, and Classification. Int J Mol Sci 2019;20. https://doi.org/10.3390/ijms20174200.

[92] Yaginuma H, Kawai S, Tabata KV, Tomiyama K, Kakizuka A, Komatsuzaki T, et al. Diversity in ATP concentrations in a single bacterial cell population revealed by quantitative single-cell imaging. Sci Rep 2014;4:6522. https://doi.org/10.1038/srep06522.

[93] Lobas MA, Tao R, Nagai J, Kronschläger MT, Borden PM, Marvin JS, et al. A genetically encoded single-wavelength sensor for imaging cytosolic and cell surface ATP. Nat Commun 2019;10:711. https://doi.org/10.1038/s41467-019-08441-5.

[94] Arai S, Kriszt R, Harada K, Looi L-S, Matsuda S, Wongso D, et al. RGB-Color Intensiometric Indicators to Visualize Spatiotemporal Dynamics of ATP in Single Cells. Angew Chem Int Ed Engl 2018;57:10873-8. https://doi.org/10.1002/anie.201804304.

[95] Nguyen PTM, Ishiwata-Kimata Y, Kimata Y. Monitoring ADP/ATP ratio in yeast cells using the fluorescent-protein reporter PercevalHR. Biosci Biotechnol Biochem 2019;83:824-8. https://doi.org/10.1080/09168451.2019.1574204.

[96] Tarasov AI, Rutter GA. Use of genetically encoded sensors to monitor cytosolic ATP/ADP ratio in living cells. Methods Enzymol 2014;542:289-311. https://doi.org/10.1016/B978-0-12-4166189.00015-7.

[97] Tantama M, Martínez-François JR, Mongeon R, Yellen G. Imaging energy status in live cells with a fluorescent biosensor of the intracellular ATP-to-ADP ratio. Nat Commun 2013;4:2550. https://doi.org/10.1038/ncomms3550.

[98] Berg J, Hung YP, Yellen G. A genetically encoded fluorescent reporter of ATP:ADP ratio. Nat Methods 2009;6:161-6. https://doi.org/10.1038/nmeth.1288.

[99] Padilla-Parra S, Tramier M. FRET microscopy in the living cell: Different approaches, strengths and weaknesses. BioEssays 2012;34:369-76. https://doi.org/10.1002/bies.201100086.

[100] Imamura H, Nhat KPH, Togawa H, Saito K, Iino R, Kato-Yamada Y, et al. Visualization of ATP levels inside single living cells with fluorescence resonance energy transfer-based genetically encoded indicators. Proc Natl Acad Sci 2009;106:15651-6. https://doi.org/10.1073/pnas.0904764106.

[101] Nakano M, Imamura H, Nagai T, Noji H. Ca ${ }^{2+}$ regulation of mitochondrial ATP synthesis visualized at the single cell level. ACS Chem Biol 2011;6:709-15. https://doi.org/10.1021/cb100313n.

[102] Douthwright S, Sluder G. Live Cell Imaging: Assessing the Phototoxicity of 488 and $546 \mathrm{~nm}$ Light and Methods to Alleviate it. J Cell Physiol 2017;232:2461-8. https://doi.org/10.1002/jcp.25588.

[103] Tsuyama T, Kishikawa J, Han Y-W, Harada Y, Tsubouchi A, Noji H, et al. In vivo fluorescent adenosine 5'-triphosphate (ATP) imaging of Drosophila melanogaster and Caenorhabditis elegans by using a genetically encoded fluorescent ATP biosensor optimized for low temperatures. Anal Chem 2013;85:7889-96. https://doi.org/10.1021/ac4015325.

[104] Zadran S, Sanchez D, Zadran H, Amighi A, Otiniano E, Wong K. Enhanced-acceptor fluorescence-based single cell ATP biosensor monitors ATP in heterogeneous cancer 
populations in real time. Biotechnol Lett 2013;35:175-80. https://doi.org/10.1007/s10529-0121065-6.

[105] Conley JM, Radhakrishnan S, Valentino SA, Tantama M. Imaging extracellular ATP with a genetically-encoded, ratiometric fluorescent sensor. PloS One 2017;12:e0187481. https://doi.org/10.1371/journal.pone.0187481.

[106] Zadran S, Standley S, Wong K, Otiniano E, Amighi A, Baudry M. Fluorescence resonance energy transfer (FRET)-based biosensors: visualizing cellular dynamics and bioenergetics. Appl Microbiol Biotechnol 2012;96:895-902. https://doi.org/10.1007/s00253-012-4449-6.

[107] Dittrich M, Hayashi S, Schulten K. On the Mechanism of ATP Hydrolysis in F1-ATPase. Biophys J 2003;85:2253-66.

[108] Kato-Yamada Y, Yoshida M. Isolated epsilon subunit of thermophilic F1-ATPase binds ATP. J Biol Chem 2003;278:36013-6. https://doi.org/10.1074/jbc.M306140200.

[109] Yagi H, Kajiwara N, Tanaka H, Tsukihara T, Kato-Yamada Y, Yoshida M, et al. Structures of the thermophilic F1-ATPase epsilon subunit suggesting ATP-regulated arm motion of its Cterminal domain in F1. Proc Natl Acad Sci U S A 2007;104:11233-8. https://doi.org/10.1073/pnas.0701045104.

[110] Iino R, Murakami T, lizuka S, Kato-Yamada Y, Suzuki T, Yoshida M. Real-time monitoring of conformational dynamics of the epsilon subunit in F1-ATPase. J Biol Chem 2005;280:40130-4. https://doi.org/10.1074/jbc.M506160200.

[111] Feniouk BA, Suzuki T, Yoshida M. The role of subunit epsilon in the catalysis and regulation of FOF1-ATP synthase. Biochim Biophys Acta 2006;1757:326-38. https://doi.org/10.1016/j.bbabio.2006.03.022.

[112] Nuskova H, Mikesova J, Efimova I, Pecinova A, Pecina P, Drahota Z, et al. Biochemical thresholds for pathological presentation of ATP synthase deficiencies. Biochem Biophys Res Commun 2020;521:1036-41. https://doi.org/10.1016/j.bbrc.2019.11.033.

[113] Kucharczyk R, Zick M, Bietenhader M, Rak M, Couplan E, Blondel M, et al. Mitochondrial ATP synthase disorders: molecular mechanisms and the quest for curative therapeutic approaches. Biochim Biophys Acta 2009;1793:186-99. https://doi.org/10.1016/j.bbamcr.2008.06.012.

[114] DiMauro S, Mancuso M. Mitochondrial diseases: therapeutic approaches. Biosci Rep 2007;27:125-37. https://doi.org/10.1007/s10540-007-9041-4.

[115] Mikol J, Polivka M. [Mitochondrial encephalomyopathies]. Ann Pathol 2005;25:282-91. https://doi.org/10.1016/s0242-6498(05)80132-4.

[116] Janssen RJRJ, van den Heuvel LP, Smeitink JAM. Genetic defects in the oxidative phosphorylation (OXPHOS) system. Expert Rev Mol Diagn 2004;4:143-56. https://doi.org/10.1586/14737159.4.2.143.

[117] Smeitink J, van den Heuvel L, DiMauro S. The genetics and pathology of oxidative phosphorylation. Nat Rev Genet 2001;2:342-52. https://doi.org/10.1038/35072063.

[118] Shepherd RK, Checcarelli N, Naini A, De Vivo DC, DiMauro S, Sue CM. Measurement of ATP production in mitochondrial disorders. J Inherit Metab Dis 2006;29:86-91. https://doi.org/10.1007/s10545-006-0148-8.

[119] Murayama K, Shimura M, Liu Z, Okazaki Y, Ohtake A. Recent topics: the diagnosis, molecular genesis, and treatment of mitochondrial diseases. J Hum Genet 2019;64:113-25. https://doi.org/10.1038/s10038-018-0528-6.

[120] Mayr JA, Haack TB, Freisinger P, Karall D, Makowski C, Koch J, et al. Spectrum of combined respiratory chain defects. J Inherit Metab Dis 2015;38:629-40. https://doi.org/10.1007/s10545015-9831-y.

[121] Frazier AE, Thorburn DR, Compton AG. Mitochondrial energy generation disorders: genes, mechanisms, and clues to pathology. J Biol Chem 2019;294:5386-95. https://doi.org/10.1074/jbc.R117.809194.

[122] Gropman AL. Diagnosis and treatment of childhood mitochondrial diseases. Curr Neurol Neurosci Rep 2001;1:185-94. https://doi.org/10.1007/s11910-001-0015-9. 
[123] Rizza T, Vazquez-Memije ME, Meschini MC, Bianchi M, Tozzi G, Nesti C, et al. Assaying ATP synthesis in cultured cells: a valuable tool for the diagnosis of patients with mitochondrial disorders. Biochem Biophys Res Commun 2009;383:58-62. https://doi.org/10.1016/j.bbrc.2009.03.121.

[124] Nasca A, Rizza T, Doimo M, Legati A, Ciolfi A, Diodato D, et al. Not only dominant, not only optic atrophy: expanding the clinical spectrum associated with OPA1 mutations. Orphanet J Rare Dis 2017;12:89. https://doi.org/10.1186/s13023-017-0641-1.

[125] Di Nottia M, Montanari A, Verrigni D, Oliva R, Torraco A, Fernandez-Vizarra E, et al. Novel mutation in mitochondrial Elongation Factor EF-Tu associated to dysplastic leukoencephalopathy and defective mitochondrial DNA translation. Biochim Biophys Acta Mol Basis Dis 2017;1863:961-7. https://doi.org/10.1016/j.bbadis.2017.01.022.

[126] Torraco A, Ardissone A, Invernizzi F, Rizza T, Fiermonte G, Niceta M, et al. Novel mutations in IBA57 are associated with leukodystrophy and variable clinical phenotypes. J Neurol 2017;264:102-11. https://doi.org/10.1007/s00415-016-8312-z.

[127] Torraco A, Verrigni D, Rizza T, Meschini MC, Vazquez-Memije ME, Martinelli D, et al. TMEM70: a mutational hot spot in nuclear ATP synthase deficiency with a pivotal role in complex V biogenesis. Neurogenetics 2012;13:375-86. https://doi.org/10.1007/s10048-012-0343-8.

[128] Martinelli D, Häberle J, Rubio V, Giunta C, Hausser I, Carrozzo R, et al. Understanding pyrroline-5-carboxylate synthetase deficiency: clinical, molecular, functional, and expression studies, structure-based analysis, and novel therapy with arginine. J Inherit Metab Dis 2012;35:761-76. https://doi.org/10.1007/s10545-011-9411-8.

[129] Ferreira M, Torraco A, Rizza T, Fattori F, Meschini MC, Castana C, et al. Progressive cavitating leukoencephalopathy associated with respiratory chain complex I deficiency and a novel mutation in NDUFS1. Neurogenetics 2011;12:9-17. https://doi.org/10.1007/s10048-010-0265-2.

[130] Blacker TS, Mann ZF, Gale JE, Ziegler M, Bain AJ, Szabadkai G, et al. Separating NADH and NADPH fluorescence in live cells and tissues using FLIM. Nat Commun 2014;5:3936. https://doi.org/10.1038/ncomms4936.

[131] Weinreb RN, Aung T, Medeiros FA. The pathophysiology and treatment of glaucoma: a review. JAMA 2014;311:1901-11. https://doi.org/10.1001/jama.2014.3192.

[132] Lu W, Hu H, Sévigny J, Gabelt BT, Kaufman PL, Johnson EC, et al. Rat, mouse, and primate models of chronic glaucoma show sustained elevation of extracellular ATP and altered purinergic signaling in the posterior eye. Invest Ophthalmol Vis Sci 2015;56:3075-83. https://doi.org/10.1167/iovs.14-15891.

[133] Stamer WD. Implications of sustained elevation in extracellular ATP in retina following chronic ocular hypertension. Invest Ophthalmol Vis Sci 2015;56:3084. https://doi.org/10.1167/iovs.1517032.

[134] Ju W-K, Kim K-Y, Lindsey JD, Angert M, Duong-Polk KX, Scott RT, et al. Intraocular pressure elevation induces mitochondrial fission and triggers OPA1 release in glaucomatous optic nerve. Invest Ophthalmol Vis Sci 2008;49:4903-11. https://doi.org/10.1167/iovs.07-1661.

[135] Zanin RF, da Silva GL, Erig T, Sperotto NDM, Leite CE, Coutinho-Silva R, et al. Decrease of serum adenine nucleotide hydrolysis in an irritant contact dermatitis mice model: potential P2X7R involvement. Mol Cell Biochem 2015;404:221-8. https://doi.org/10.1007/s11010-015-23817.

[136] Lee HY, Stieger M, Yawalkar N, Kakeda M. Cytokines and chemokines in irritant contact dermatitis. Mediators Inflamm 2013;2013:916497. https://doi.org/10.1155/2013/916497.

[137] Mizumoto N, Mummert ME, Shalhevet D, Takashima A. Keratinocyte ATP Release Assay for Testing Skin-Irritating Potentials of Structurally Diverse Chemicals. J Invest Dermatol 2003;121:1066-72. https://doi.org/10.1046/j.1523-1747.2003.12558.x.

[138] Eggers C, Arendt G, Hahn K, Husstedt IW, Maschke M, Neuen-Jacob E, et al. HIV-1-associated neurocognitive disorder: epidemiology, pathogenesis, diagnosis, and treatment. J Neurol 2017;264:1715-27. https://doi.org/10.1007/s00415-017-8503-2. 
[139] Antinori A, Arendt G, Becker JT, Brew BJ, Byrd DA, Cherner M, et al. Updated research nosology for HIV-associated neurocognitive disorders. Neurology 2007;69:1789-99. https://doi.org/10.1212/01.WNL.0000287431.88658.8b.

[140] Boerwinkle A, Ances BM. Molecular Imaging of Neuroinflammation in HIV. J Neuroimmune Pharmacol Off J Soc NeuroImmune Pharmacol 2019;14:9-15. https://doi.org/10.1007/s11481-0189823-4.

[141] Velasquez S, Prevedel L, Valdebenito S, Gorska AM, Golovko M, Khan N, et al. Circulating levels of ATP is a biomarker of HIV cognitive impairment. EBioMedicine 2020;51:102503. https://doi.org/10.1016/j.ebiom.2019.10.029.

[142] Gendelman HE. Predictive biomarkers for cognitive decline during progressive HIV infection. EBioMedicine 2020;51:102538. https://doi.org/10.1016/j.ebiom.2019.10.064.

[143] Guth CM, Luo W, Jolayemi O, Chadalavada KS, Komalavilas P, Cheung-Flynn J, et al. Adenosine triphosphate as a molecular mediator of the vascular response to injury. J Surg Res 2017;216:80-6. https://doi.org/10.1016/j.jss.2017.03.025.

[144] Zhang H, Shen Z, Hogan B, Barakat AI, Misbah C. ATP Release by Red Blood Cells under Flow: Model and Simulations. Biophys J 2018;115:2218-29. https://doi.org/10.1016/j.bpj.2018.09.033.

[145] Yeung PK, Kolathuru SS, Mohammadizadeh S, Akhoundi F, Linderfield B. Adenosine 5'Triphosphate Metabolism in Red Blood Cells as a Potential Biomarker for Post-Exercise Hypotension and a Drug Target for Cardiovascular Protection. Metabolites 2018;8. https://doi.org/10.3390/metabo8020030.

[146] Li Y, Zhou J, Burkovskiy I, Yeung P, Lehmann C. ATP in red blood cells as biomarker for sepsis in humans. Med Hypotheses 2019;124:84-6. https://doi.org/10.1016/j.mehy.2019.02.014.

[147] Kujawa J, Zavodnik L, Zavodnik I, Buko V, Lapshyna A, Bryszewska M. Effect of LowIntensity (3.75-25 J/cm2) Near-Infrared (810 nm) Laser Radiation on Red Blood Cell ATPase Activities and Membrane Structure. J Clin Laser Med Surg 2004;22:111-7. https://doi.org/10.1089/104454704774076163.

[148] Kilańczyk E, Pałecz D, Bryszewska M. Effect of Red Laser Light on Na+,K+-ATPase Activity in Human Erythrocyte Membranes Sensitized with Zn-Phthalocyanine. J Clin Laser Med Surg 2002;20:71-5. https://doi.org/10.1089/104454702753768043.

[149] Clausen MV, Hilbers F, Poulsen H. The Structure and Function of the Na,K-ATPase Isoforms in Health and Disease. Front Physiol 2017;8. https://doi.org/10.3389/fphys.2017.00371.

[150] Suardi N, Sodipo BK, Mustafa MZ, Ali Z. Effect of visible laser light on ATP level of anaemic red blood cell. J Photochem Photobiol B 2016;162:703-6. https://doi.org/10.1016/j.jphotobiol.2016.07.041.

[151] Yuan X, Song Y, Song Y, Xu J, Wu Y, Glidle A, et al. Effect of Laser Irradiation on Cell Function and Its Implications in Raman Spectroscopy. Appl Environ Microbiol 2018;84. https://doi.org/10.1128/AEM.02508-17.

[152] Farthing DE, Farthing CA, Xi L. Inosine and hypoxanthine as novel biomarkers for cardiac ischemia: from bench to point-of-care. Exp Biol Med Maywood NJ 2015;240:821-31. https://doi.org/10.1177/1535370215584931.

[153] Deli T, Csernoch L. Extracellular ATP and cancer: an overview with special reference to P2 purinergic receptors. Pathol Oncol Res POR 2008;14:219-31. https://doi.org/10.1007/s12253-0089071-7.

[154] Seyfried TN, Arismendi-Morillo G, Mukherjee P, Chinopoulos C. On the Origin of ATP Synthesis in Cancer. IScience 2020;23:101761. https://doi.org/10.1016/j.isci.2020.101761.

[155] Wallace DC. Mitochondria and cancer. Nat Rev Cancer 2012;12:685-98. https://doi.org/10.1038/nrc3365.

[156] Di Virgilio F, Sarti AC, Falzoni S, De Marchi E, Adinolfi E. Extracellular ATP and P2 purinergic signalling in the tumour microenvironment. Nat Rev Cancer 2018;18:601-18. https://doi.org/10.1038/s41568-018-0037-0. 
[157] Qian Y, Wang X, Li Y, Cao Y, Chen X. Extracellular ATP a New Player in Cancer Metabolism: NSCLC Cells Internalize ATP In Vitro and In Vivo Using Multiple Endocytic Mechanisms. Mol Cancer Res MCR 2016;14:1087-96. https://doi.org/10.1158/1541-7786.MCR-16-0118.

[158] Vultaggio-Poma V, Sarti AC, Di Virgilio F. Extracellular ATP: A Feasible Target for Cancer Therapy. Cells 2020;9. https://doi.org/10.3390/cells9112496.

[159] Wu W, Zhao S. Metabolic changes in cancer: beyond the Warburg effect. Acta Biochim Biophys Sin 2013;45:18-26. https://doi.org/10.1093/abbs/gms104.

[160] Pascale RM, Calvisi DF, Simile MM, Feo CF, Feo F. The Warburg Effect 97 Years after Its Discovery. Cancers 2020;12. https://doi.org/10.3390/cancers12102819.

[161] McCafferty CE, Abi-Hanna D, Aghajani MJ, Micali GT, Lockart I, Vickery K, et al. The validity of adenosine triphosphate measurement in detecting endoscope contamination. J Hosp Infect 2018;100:e142-5. https://doi.org/10.1016/j.jhin.2018.08.004.

[162] Sanna T, Dallolio L, Raggi A, Mazzetti M, Lorusso G, Zanni A, et al. ATP bioluminescence assay for evaluating cleaning practices in operating theatres: applicability and limitations. BMC Infect Dis 2018;18:583. https://doi.org/10.1186/s12879-018-3505-y.

[163] Nante N, Ceriale E, Messina G, Lenzi D, Manzi P. Effectiveness of ATP bioluminescence to assess hospital cleaning: a review. J Prev Med Hyg 2017;58:E177-83.

[164] Watanabe A, Tamaki N, Yokota K, Matsuyama M, Kokeguchi S. Use of ATP bioluminescence to survey the spread of aerosol and splatter during dental treatments. J Hosp Infect 2018;99:303-5. https://doi.org/10.1016/j.jhin.2018.03.002.

[165] Okanojo M, Miyashita N, Tazaki A, Tada H, Hamazoto F, Hisamatsu M, et al. Attomol-level ATP bioluminometer for detecting single bacterium. Lumin J Biol Chem Lumin 2017;32:751-6. https://doi.org/10.1002/bio.3246.

[166] Brodl E, Winkler A, Macheroux P. Molecular Mechanisms of Bacterial Bioluminescence. Comput Struct Biotechnol J 2018;16:551-64. https://doi.org/10.1016/j.csbj.2018.11.003.

[167] Kim SU, Jo E-J, Noh Y, Mun H, Ahn Y-D, Kim M-G. Adenosine Triphosphate BioluminescenceBased Bacteria Detection Using Targeted Photothermal Lysis by Gold Nanorods. Anal Chem 2018;90:10171-8. https://doi.org/10.1021/acs.analchem.8b00254. 